\title{
ASYMPTOTIC ANALYSIS OF GAUSSIAN INTEGRALS. I. ISOLATED MINIMUM POINTS
}

\author{
BY \\ RICHARD S. ELLIS ${ }^{1}$ AND JAY S. ROSEN ${ }^{2}$
}

\begin{abstract}
This paper derives the asymptotic expansions of a wide class of Gaussian function space integrals under the assumption that the minimum points of the action are isolated. Degenerate as well as nondegenerate minimum points are allowed. This paper also derives limit theorems for related probability measures which correspond roughly to the law of large numbers and the central limit theorem. In the degenerate case, the limits are non-Gaussian.
\end{abstract}

I. Introduction. Function space integrals are useful in many areas of mathematics and physics. Physical problems often give rise to Gaussian function space integrals depending on a parameter and the asymptotics with respect to the parameter yield important information about the original problem. This paper is the first in a series of papers on the asymptotic expansions of a wide class of Gaussian function space integrals and on limit theorems for related probability measures. This paper treats the case where the minimum points of the action are isolated. Previous work on this case [Schilder, Pincus] has assumed a nondegeneracy condition which assures that one never strays too far from the realm of Gaussian measures. Our results cover both the nondegenerate case and the degenerate case, the analysis of the latter being much more subtle. In the degenerate case, the leading asymptotic behavior is non-Gaussian. The second paper in this series treats the case where the minimum points of the action form a smooth manifold. This case plays an important role in recent physics [Coleman, Wiegel]. Subsequent papers will treat applications. The results in the present paper were inspired by our work on a model in statistical mechanics [Ellis-Rosen (1),(2)]. As background, we cite the important papers of [Varadhan] and [Donsker-Varadhan].

This paper and the sequel consider Gaussian integrals on a real separable Hilbert space. While certain Banach spaces are important in applications, the Hilbert space setting leads to simplifications in the statements and proofs of the theorems. By using the results of [Kallianpur-Oodaira], it is not hard to generalize our results to abstract Wiener spaces.

Received by the editors March 11, 1981.

1980 Mathematics Subject Classification. Primary 28C20, 58C99, 60B10, 60F05.

Key words and phrases. Asymptotic expansion, Gaussian integral, nondegenerate minimum point, simply degenerate minimum point, multidegenerate minimum point.

'Alfred P. Sloan Research Fellow. Research supported in part by NSF Grant MCS-80-02149.

${ }^{2}$ Research supported in part by NSF Grant MCS-80-02140. 
Let $P_{A}$ be a mean zero Gaussian probability measure with covariance operator $A$ on a real separable Hilbert space $\mathcal{H}$. The inner product and norm of $\mathcal{H}$ are denoted by $\langle-,-\rangle$ and $\|-\|$, respectively. Let $\psi$ and $F$ be real-valued $C^{\infty}$-Fréchet differentiable functionals on $\mathcal{H}$ which satisfy the bounds $(2.1)(\mathrm{a})-(\mathrm{b})$. We study the asymptotics of

$$
J_{n}:=\int \psi(Y / \sqrt{n}) e^{-n F\left(Y /{ }^{n} n\right)} d P_{A}(Y)
$$

as $n \rightarrow \infty$. Here and below, all integrations extend over $\mathcal{H}$ unless otherwise noted.

Let $f$ and $g$ be smooth, real-valued functions on $\mathbf{R}$ such that $g(x) \rightarrow \infty$ sufficiently fast as $x \rightarrow \infty$. Then Laplace's method tells us that the asymptotics of $\int_{\mathbf{R}} f(x) \exp (-n g(x)) d x$ are determined by the behavior of $g$ near its minimum points [Erdelyi, §2.4]. Formally, $J_{n}$ in (1.1) can be written as

$$
\begin{aligned}
\int \psi(Y / \sqrt{n}) \exp (-n F & \left.(Y / \sqrt{n})-\left\langle A^{-1} Y, Y\right\rangle / 2\right) d Y \\
& =\int \psi(Y / \sqrt{n}) \exp (-n G(Y / \sqrt{n})) d Y,
\end{aligned}
$$

where $G(Y):=F(Y)+\left\langle A^{-1} Y, Y\right\rangle / 2 . G$ is called the (Euclidean) action. By analogy with the situation on $\mathbf{R}$, we expect the asymptotics of $J_{n}$ to be determined by the behavior of $G$ near its minimum points. The expressions in (1.2) are purely formal (unless $\operatorname{dim} \mathcal{H}<\infty$ ) since the symbol $d Y$ is supposed to represent the nonexistent translation invariant measure on $\mathcal{H}$. Also, $\left\langle A^{-1} Y, Y\right\rangle / 2=\left\langle A^{-1 / 2} Y, A^{-1 / 2} Y\right\rangle / 2$ is defined only for $Y \in \mathscr{D}\left(A^{-1 / 2}\right)$ which in general is only a dense subset of $\mathcal{H}$.

We define $G^{*}:=\inf G$ on $\mathcal{H}$ and assume for now that $G$ has a unique minimum point $Y^{*}$. Thus, $G\left(Y^{*}\right)=G^{*}$. We necessarily have

$$
G^{\prime}\left(Y^{*}\right)=0, \quad G^{\prime \prime}\left(Y^{*}\right) \geqslant 0 \text { on } \mathcal{H} \text {. }
$$

Here, $G^{\prime}\left(Y^{*}\right)$ and $G^{\prime \prime}\left(Y^{*}\right)$ are respectively the element of $\mathcal{H}$ and the linear operator on $\mathcal{H}$ defined by the first and second Fréchet derivatives of $G$ at $Y^{*}$. We set $\mathscr{K}:=\operatorname{ker}\left(G^{\prime \prime}\left(Y^{*}\right)\right)$. Because of the compactness of $A$, we have in general $\operatorname{dim} \mathscr{K}<\infty$ (see after (3.8) in $\S \mathrm{III})$. We say that $Y^{*}$ is nondegenerate if $\mathscr{K}=\{0\}$, simply degenerate if $\operatorname{dim} \mathscr{K}=1$, and multidegenerate if $\operatorname{dim} \mathscr{K}>1$. This paper treats the first two cases in detail. As we point out below in Remark 2.4(c), the multidegenerate case can be treated exactly like the simply degenerate case provided an extra integrability condition is satisfied. However, if the condition fails, then our methods do not apply.

Theorem 2.1 states the asymptotic expansion of $\exp \left(n G^{*}\right) J_{n}$ in the nondegenerate case. We find

$$
e^{n G^{*}} J_{n} \sim \sum_{j \geqslant 0} \Gamma_{j} n^{-j}
$$

where the $\left\{\Gamma_{j}\right\}$ are functionals, explicitly computable in terms of $A$ and the Fréchet derivatives of $\psi$ and $F$ at $Y^{*}$. Here and below, an expression such as (1.4) means that for any $i \in\{0,1,2, \ldots\}$

$$
\lim _{n \rightarrow \infty} n^{i}\left(e^{n G^{*}} J_{n}-\sum_{j=0}^{i} \Gamma_{j} n^{-j}\right)=0 .
$$


The leading order term in (1.4) is $\Gamma_{0}:=\Delta^{-1 / 2} \psi\left(Y^{*}\right)$, where $\Delta$ is a specific determinant defined in Theorem 2.1. In the special case of Wiener measure, Theorem 2.1 was proved by [Schilder]. Theorem 2.3 states the asymptotic expansion in the simply degenerate case. We find

$$
e^{n G^{*}} J_{n} \sim n^{1 / 2-1 / k} \sum_{j \geqslant 0} \Gamma_{j, k} n^{-2 j / k} .
$$

Here $k$ is an even integer exceeding 2 which measures the extent of the degeneracy of $G$ at $Y^{*} ; k$ is called the type of $Y^{*}$. The definition of $k$ is a nontrivial matter involving a nonlinear change of coordinates in a neighborhood of $Y^{*}$. We consider the definition of type to be one of the main contributions of this paper. In (1.5), the $\left\{\Gamma_{j, k}\right\}$ are functionals, explicitly computable in terms of $A$ and the Fréchet derivatives of $\psi$ and $F$ at $Y^{*}$. The leading order term in (1.5) is $n^{1 / 2-1 / k} C \tilde{\Delta}^{-1 / 2} \psi\left(Y^{*}\right)$, where $C$ is an explicit constant and $\tilde{\Delta}$ a specific determinant defined in Theorem 2.3.

Our other results are limit theorems for probability measures related to $J_{n}$. We define the probability measures

$$
d Q_{n}(Y):=\frac{\exp (-n F(Y / n)) d P_{A}(Y / \sqrt{n})}{Z_{n}},
$$

where $Z_{n}$ is the normalization constant

$$
Z_{n}:=\int \exp (-n F(Y / \sqrt{n})) d P_{A}(Y)
$$

Let $Y_{n}$ be an $\mathcal{H}$-valued random variable with distribution $Q_{n}$. The scaling in the definition of $Q_{n}$ has been chosen in order to suggest the analogy between the asymptotic behavior of $\left\{Y_{n}\right\}$ and the law of large numbers and the central limit theorem. If $F \equiv 0$, then the analogy is exact, for in this case $Y_{n}$ is distributed like the sum of $n$ i.i.d. random variables, each distributed by $P_{A}$. It is easy to see that

$$
\text { (a) } Y_{n} / n \stackrel{\mathscr{D}}{\rightarrow} \delta_{0}, \quad \text { (b) } Y_{n} / \sqrt{n} \stackrel{\mathscr{D}}{=} P_{A} \text {. }
$$

Here, $\delta_{Y}$ denotes the point mass at $Y$ and $\stackrel{\mathscr{D}}{\rightarrow}$ denotes convergence in distribution. For general $F$, the asymptotic behavior of $\left\{Y_{n}\right\}$ is much more complicated. Suppose for simplicity (and only in this paragraph) that $Y^{*}=0$ is the unique minimum point of $G$. Then (1.8)(a) stays valid (Theorem 2.5). We prove in Theorem 2.6 that if $Y^{*}=0$ is nondegenerate, then

$$
Y_{n} / \sqrt{n} \stackrel{\mathscr{D}}{\rightarrow} P_{B}
$$

where $P_{B}$ is an explicitly determined Gaussian measure. We prove in Theorem 2.7 that if $Y^{*}=0$ is simply degenerate of type $k$, then there exists a non-Gaussian probability measure $\zeta$ on $\mathcal{K}$, concentrated on the one-dimensional subspace $\mathscr{K}:=\operatorname{ker}\left(G^{\prime \prime}\left(Y^{*}\right)\right)$, such that

$$
Y_{n} / n^{1-1 / k} \stackrel{\mathscr{D}}{\rightarrow} \zeta
$$


We may think of (1.10) as a breakdown of the central limit theorem (since $k \geqslant 4$ ) due to high correlations in $Y_{n}$ along the degenerate subspace $\mathcal{K}$. In contrast to the $F \equiv 0$ case, for general $F, Y_{n}$ is not distributed like the sum of $n \mathcal{F}$-valued random variables. However, we showed in [Ellis-Rosen (1)] the close connection between $Y_{n}$ and the sum of $n$ dependent random variables on $\mathbf{R}$ for a special choice of $F$ and $P_{A}$ in (1.6). This sum is an important quantity in a model in statistical mechanics. We used the analogues of (1.9)-(1.10) to deduce the asymptotic behavior of this sum and thus to obtain useful information about the model. For example, $(1.10)$ is related to a phase transition.

In §II of this paper we state our main theorems. §III derives a number of facts needed for the proofs of the theorems. §IV proves the asymptotic expansion (1.4) (Theorem 2.1) and the limit theorem (1.9) (Theorem 2.6) in the nondegenerate case. In addition Theorem 4.6 extends the limit theorems (1.8)(a) and (1.9) to the case where $G$ has nonunique minimum points, all of which are nondegenerate. (By Theorem 3.2, nondegenerate minimum points must be isolated.) $\S \mathrm{V}$ proves the asymptotic expansion (1.5) (Theorem 2.3) and the limit theorem (1.10) (Theorem 2.7) in the simply degenerate case. In addition, Theorem 5.5 extends the limit theorems (1.8)(a) and (1.10) to the case where $G$ has nonunique isolated minimum points, of which some are simply degenerate and the rest nondegenerate.

II. Statement of results. Since the support of $P_{A}$ equals the closure of the range of $A$ [Rajput], we can assume without loss of generality that $A>0$. Throughout this paper we assume that $\psi$ and $F$ are real-valued $C^{\infty}$ Fréchet differentiable functionals on $\mathcal{H}$ and that there exist constants $b_{1}>0, b_{2} \geqslant 0,0 \leqslant b_{3}<1 /(2\|A\|), b_{4} \geqslant 0$ such that
(a) $|\psi(Y)| \leqslant b_{1} \exp \left(b_{2}\|Y\|^{2}\right)$,
(b) $F(Y) \geqslant-b_{3}\|Y\|^{2}-b_{4}$

for $Y \in \mathcal{H}$. These bounds are sufficient to assure that $J_{n}$ exists. Our theorems go through if $(2.1)(b)$ is replaced by certain weaker lower bounds which arise naturally in applications [Simon, §18].

Throughout this section we assume that $G$ has a unique minimum point $Y^{*}$. Our first two results, Theorems 2.1 and 2.3 , state the asymptotic expansion of $\exp \left(n G^{*}\right) J_{n}$ in the case that $Y^{*}$ is nondegenerate and the case that $Y^{*}$ is simply degenerate, respectively. In Remark 2.4 (c) we briefly discuss the multidegenerate case. If $G$ has nonunique, isolated minimum points which are all either nondegenerate or simply degenerate, then the asymptotic expansion is obtained by adding the contributions made by each minimum point. The contribution of a minimum point is given by (2.4) if it is nondegenerate and by (2.20) if it is simply degenerate. Since isolated minimum points must be finite in number (Lemma 3.1), this procedure is well defined. By Theorem 3.2 all nondegenerate minimum points are isolated.

Before stating Theorem 2.1, we give the intuition behind it. Recall that $Y^{*}$ is nondegenerate if $\mathcal{K}:=\operatorname{ker}\left(G^{\prime \prime}\left(Y^{*}\right)\right)=\{0\}$. Given such a $Y^{*}$, we can write for $\|Y\|$ small

$$
G\left(Y^{*}+Y\right)=G\left(Y^{*}\right)+\frac{1}{2}\left\langle G^{\prime \prime}\left(Y^{*}\right) Y, Y\right\rangle+\text { error terms. }
$$


(See (2.3) for an explicit formula for the error terms.) Thus, the important term in the exponent of (1.2) is $\frac{1}{2}\left\langle G^{\prime \prime}\left(Y^{*}\right) Y, Y\right\rangle$. Since $G^{\prime \prime}\left(Y^{*}\right)$ is invertible (a consequence of the nondegeneracy), this term gives rise to a Gaussian measure with covariance operator $\left[G^{\prime \prime}\left(Y^{*}\right)\right]^{-1}$. The asymptotics of $\exp \left(n G^{*}\right) J_{n}$ are derived by expanding around this measure. It is not hard to show that the error terms in (2.2) are given by the functional

$$
F_{3}(Y):=F\left(Y^{*}+Y\right)-F\left(Y^{*}\right)-\left\langle F^{\prime}\left(Y^{*}\right), Y\right\rangle-\frac{1}{2}\left\langle F^{\prime \prime}\left(Y^{*}\right) Y, Y\right\rangle .
$$

$F_{3}$ enters the asymptotic expansion in Theorem 2.1 below.

THEOREM 2.1. Suppose that $G$ has a unique minimum point $Y^{*}$ and that $Y^{*}$ is nondegenerate. Then $B:=\left[G^{\prime \prime}\left(Y^{*}\right)\right]^{-1}$ exists and is the covariance operator of a mean zero Gaussian measure $P_{B}$ on $\mathcal{H}$, and $\exp \left(n G^{*}\right) J_{n}$ has the asymptotic expansion (see the explanation below)

$$
e^{n G^{*}} J_{n} \sim \Delta^{-1 / 2} \int \psi\left(Y^{*}+Y / \sqrt{n}\right) \exp \left[-n F_{3}(Y / \sqrt{n})\right] d P_{B}(Y) \text { as } n \rightarrow \infty \text {. }
$$

In (2.4), $\Delta:=\operatorname{det}\left(I+A F^{\prime \prime}\left(Y^{*}\right)\right), \Delta$ is well defined and $\Delta>0$. The leading term in (2.4) equals $\Delta^{-1 / 2} \psi\left(Y^{*}\right)$.

Explanation. Because of the smoothness of $\psi$ and $F$, the integrand on the right-hand side of (2.4) has for each $Y \in \mathcal{H}$ an asymptotic expansion of the form

$$
\Delta^{-1 / 2} \psi\left(Y^{*}+Y / \sqrt{n}\right) \exp \left[-n F_{3}(Y / \sqrt{n})\right] \sim \sum_{j \geqslant 0} a_{j}(Y) n^{-j / 2} \text { as } n \rightarrow \infty,
$$

where the $\left\{a_{i}(Y)\right\}$ are functionals. Then (2.4) means that $\exp \left(n G^{*}\right) J_{n}$ has the asymptotic expansion

$$
e^{n G^{*} J_{n}} \sim \sum_{j \geqslant 0}\left(\int a_{j}(Y) d P_{B}(Y)\right) n^{-j / 2} \text { as } n \rightarrow \infty .
$$

For $j$ odd, $a_{j}(Y)$ turns out to be an odd function of $Y$. Since $P_{B}$ is mean zero, the expansion (2.4) has the form (1.4); i.e., only integral powers of $n^{-1}$ appear.

REMARK 2.2. (a) We give the first two terms in (2.4) if $\psi \equiv 1$ :

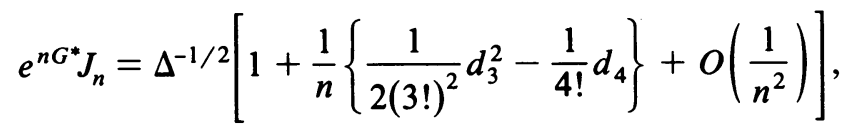

where $d_{j}:=\int D^{j} F\left(Y^{*}\right) Y^{j} d P_{B}(Y)$. For details, see the proof of Theorem 2.1.

(b) In (2.4), we multiply $J_{n}$ by $\exp \left(n G^{*}\right)$ in order to cancel the contribution of $G\left(Y^{*}\right)=G^{*}$ when $(2.2)$ is substituted into (1.2). This also applies to the simply degenerate case.

We now discuss the asymptotic expansion in the simply degenerate case. Recall that $Y^{*}$ is simply degenerate if $\operatorname{dim} \mathscr{K}=1$. Given such a $Y^{*}$, the previous analysis completely breaks down since $G^{\prime \prime}\left(Y^{*}\right)$ is no longer invertible and we can no longer expand around a Gaussian measure. Let $\tau$ denote the orthogonal projection onto $\mathcal{K}^{\perp}$ and pick $U \in \mathscr{K}$ a unit vector. (One easily checks that all of our results are independent of the sign of $U$.) We write $Y \in \mathcal{H}$ in the form

$$
Y=z U+X \in \mathscr{K} \oplus \mathcal{K}^{\perp},
$$


where $z:=\langle Y, U\rangle$ and $X:=\tau Y$. Following (2.2), we examine $G\left(Y^{*}+Y\right)$ for $\|Y\|$ small. Writing $Y$ as in (2.6), we find for some $m>2$

$$
\begin{aligned}
G\left(Y^{*}+Y\right)= & G\left(Y^{*}\right)+\frac{z^{m}}{m !} D^{m} G\left(Y^{*}\right) U^{m}+\frac{1}{2}\left\langle G^{\prime \prime}\left(Y^{*}\right) X, X\right\rangle \\
& +(\text { cross terms in } z, X)+(\text { error term in } z, X) .
\end{aligned}
$$

The cross terms are all terms which are of order $O\left(z^{j}\|X\|\right)$ for $j \in\{1,2, \ldots, m-1\}$ while the error terms are all terms which are of order $o\left(z^{m}\right)$ or $o\left(\|X\|^{2}\right)$. Unfortunately, when inserted into (1.2), the cross terms (2.7) contribute to the leading asymptotics of $J_{n}$. We introduce a new way of writing $Y$ which eliminates these cross terms altogether.

We modify (2.6) by writing $Y$ in the form

$$
Y=z U+\Phi(z U)+X,
$$

where $z:=\langle Y, U\rangle$ and $X \in \mathcal{K}^{\perp}$. For each $V \in \mathcal{H}$ with $\|V\|$ sufficiently small (in particular, for $V:=z U, z$ real and sufficiently small) $\Phi(V)$ is an element of $\mathcal{H}$ to be defined explicitly in (2.11) below. The map $\Phi: V \rightarrow \Phi(V)$ is $C^{\infty}$ and satisfies $\Phi(0)=0$. These properties imply that $\|\Phi(z U)\|=O(z)$ as $|z| \rightarrow 0$. Hence, we find that for $\|Y\|$ small,

$$
\begin{aligned}
G\left(Y^{*}+Y\right)= & G\left(Y^{*}+z U+\Phi(z U)+X\right) \\
= & G\left(Y^{*}+z U+\Phi(z U)\right)+\left\langle G^{\prime}\left(Y^{*}+z U+\Phi(z U)\right), X\right\rangle \\
& +\frac{1}{2}\left\langle G^{\prime \prime}\left(Y^{*}+z U+\Phi(z U)\right) X, X\right\rangle+o\left(\|X\|^{2}\right) \\
= & G\left(Y^{*}+z U+\Phi(z U)\right)+\left\langle G^{\prime}\left(Y^{*}+z U+\Phi(z U)\right), X\right\rangle \\
& +\frac{1}{2}\left\langle G^{\prime \prime}\left(Y^{*}\right) X, X\right\rangle+o\left(\|X\|^{2}\right) .
\end{aligned}
$$

The idea is to pick $\Phi(z U)$ in order to eliminate the cross terms; i.e., to make

$$
\left\langle G^{\prime}\left(Y^{*}+z U+\Phi(z U)\right), X\right\rangle=0 \text { for all } X \in \mathscr{K}^{\perp} \text {. }
$$

Since $Y^{*}$ is simply degenerate, the operator $\tau G^{\prime \prime}\left(Y^{*}\right) \tau$ is invertible on $\mathcal{K}^{\perp}$ with inverse operator denoted by $B_{\perp}$. By the implicit function theorem, the relation

$$
\Phi=-B_{\perp} \tau F_{3}^{\prime}(V+\Phi)
$$

defines a unique $C^{\infty} \mathcal{K}^{\perp}$-valued function $\Phi(V)$ for all $V \in \mathcal{H}$ of sufficiently small norm. The functional $F_{3}$ in (2.11) was defined in (2.3). In Lemma 5.2, we prove that with this $\Phi(2.10)$ holds. $\Phi(z U)$ can be computed to any order in $z$ by a straightforward iteration.

We now turn to the leading order behavior in $z$ of $G\left(Y^{*}+Y\right)$ in (2.9). Suppose that for small $z$ we can write

$$
G\left(Y^{*}+z U+\Phi(z U)\right)=G\left(Y^{*}\right)+\lambda z^{k}+O\left(z^{k+1}\right),
$$

where $\lambda=\lambda\left(Y^{*}\right)$ is nonzero and $k=k\left(Y^{*}\right)$ is a positive integer. We call $k$ the type and $\lambda$ the strength of $Y^{*}$. (If there exists no integer $k$ such that the expansion (2.12) is valid for small $z$, then we define $k\left(Y^{*}\right)=\infty$ and $\lambda\left(Y^{*}\right)=0$.) We have explicitly

$$
\begin{gathered}
k=\min \left\{j: j \in\{1,2, \ldots\}, d^{j} G\left(Y^{*}+z U+\Phi(z U)\right) /\left.d z^{j}\right|_{z=0} \neq 0\right\}, \\
\lambda=d^{k} G\left(Y^{*}+z U+\Phi(z U)\right) /\left.d z^{k}\right|_{z=0} .
\end{gathered}
$$


Since $Y^{*}$ is an isolated, simply degenerate minimum point, we must have $\lambda>0, k$ even, and one can show $k \geqslant 4$ (Lemma 4.3). Since $k$ is even, the definitions of type and strength are independent of the sign of $U$.

From (2.9), (2.10), and (2.12), we finally have the correct analogue of (2.2) in the simply degenerate case:

$$
\begin{aligned}
G\left(Y^{*}+Y\right) & =G\left(Y^{*}+z U+\Phi(z U)+X\right) \\
& =G\left(Y^{*}\right)+\lambda z^{k}+\frac{1}{2}\left\langle G^{\prime \prime}\left(Y^{*}\right) X, X\right\rangle+(\text { error terms in } z, X) \\
& =G\left(Y^{*}\right)+\lambda z^{k}+\frac{1}{2}\left\langle\tau G^{\prime \prime}\left(Y^{*}\right) \tau X, X\right\rangle+(\text { error terms in } z, X),
\end{aligned}
$$

where the error terms are of order $O\left(z^{k+1}\right)$ and $o\left(\|X\|^{2}\right)$. (See (2.18) for an explicit formula for the error terms.) By (2.15), we see that $G$ approximately decouples along the subspaces $\mathscr{K}$ and $\mathcal{K}^{\perp}$. This decoupling gives rise in (1.2) to a non-Gaussian measure $\exp \left(-\lambda z^{k}\right) d z$ in the degenerate subspace $\mathscr{K}$ and a Gaussian measure with covariance operator $\left(\tau G^{\prime \prime}\left(Y^{*}\right) \tau\right)^{-1}$ in the nondegenerate subspace $\mathcal{K}^{\perp}$. The asymptotics of $\exp \left(n G^{*}\right) J_{n}$ are obtained by expanding around these two measures in the respective subspaces.

We need several definitions in order to simplify the statement of Theorem 2.3. For $z$ real and sufficiently small, we define

$$
W(z):=z U+\Phi(z U) .
$$

Since $\Phi(z U)$ is well defined and smooth for all real, sufficiently small $z, W(z)$ shares these properties. For $W \in \mathcal{H}$ and $X \in \mathcal{K}^{\perp}$, we define the functional

$$
\begin{aligned}
\tilde{F}_{3}(W, X):= & F\left(Y^{*}+W+X\right)-F\left(Y^{*}+W\right) \\
& -\left\langle F^{\prime}\left(Y^{*}+W\right), X\right\rangle-\frac{1}{2}\left\langle F^{\prime \prime}\left(Y^{*}\right) X, X\right\rangle .
\end{aligned}
$$

This functional enters the expression for the error terms in $z, X$ which appear in the expansion (2.15). These error terms are given by

$$
G\left(Y^{*}+W(z)\right)-G\left(Y^{*}\right)-\lambda z^{k}+\tilde{F}_{3}(W(z), X) .
$$

(By (2.12) the first three terms of $(2.18)$ are $O\left(z^{k+1}\right)$; by $(5.23) \tilde{F}_{3}(W(z), X)=$ $o\left(\|X\|^{2}\right)$ as $|z|+\|X\| \rightarrow 0$.) The functional $\tilde{F}_{3}$ is the analogue in the simply degenerate case of the functional $F_{3}$ defined in (2.3). Finally, for $z$ real and sufficiently small and $X \in \mathscr{K}^{\perp}$, we define

$$
\begin{aligned}
\Omega_{n}(z, X):= & \psi\left(Y^{*}+W(z)+X\right) \\
& \cdot \exp \left[-n\left\{G\left(Y^{*}+W(z)\right)-G^{*}-\lambda z^{k}\right\}\right] \exp \left[-n \tilde{F}_{3}(W(z), X)\right] .
\end{aligned}
$$

THEOREM 2.3. Suppose that $G$ has a unique minimum point $Y^{*}$ and that $Y^{*}$ is simply degenerate. Then $\tau G^{\prime \prime}\left(Y^{*}\right) \tau$ is invertible on $\mathcal{K}^{\perp}$ with inverse operator denoted by $B_{\perp}$. $B_{\perp}$ is the covariance operator of a mean zero Gaussian measure $P_{B_{\perp}}$ on $\mathcal{K}^{\perp}$. Suppose further that $Y^{*}$ has type $k<\infty$ and strength $\lambda$. Then $\exp \left(n G^{*}\right) J_{n}$ has the asymptotic expansion (see the explanation below)

$$
e^{n G^{*}} J_{n} \sim n^{1 / 2-1 / k}[2 \pi\langle A U, U\rangle \tilde{\Delta}]^{-1 / 2} \int_{\mathbf{R} \times \mathcal{K}^{\perp}} \Omega_{n}\left(z / n^{1 / k}, X / \sqrt{n}\right) e^{-\lambda z^{k}} d z d P_{B_{\perp}}(X)
$$


In (2.20), $\tilde{\Delta}:=\operatorname{det}\left(I+\tau \rho A \rho \tau F^{\prime \prime}\left(Y^{*}\right) \tau\right)$, where $\rho$ is the bounded operator on $\mathcal{H}$ defined by $\rho Y:=Y-[\langle A U, Y\rangle /\langle A U, U\rangle] U ; \tilde{\Delta}$ is well defined and $\tilde{\Delta}>0$. The leading term in (2.20) is

$$
n^{1 / 2-1 / k}[2 \pi\langle A U, U\rangle \tilde{\Delta}]^{-1 / 2} \int_{\mathbf{R}} e^{-\lambda z^{k}} d z \psi\left(Y^{*}\right) .
$$

Explanation. The smoothness of $\psi$ and $F$ and the fact that $W(z)$ is well defined and smooth for real sufficiently small $z$ imply that for each $z \in \mathbf{R}, X \in \mathscr{K}^{\perp}$, the integrand on the right-hand side of (2.20) has an asymptotic expansion of the form

$$
n^{1 / 2-1 / k}[2 \pi\langle A U, U\rangle \tilde{\Delta}]^{-1 / 2} \Omega_{n}\left(z / n^{1 / k}, X / \sqrt{n}\right) \sim n^{1 / 2-1 / k} \sum_{j \geqslant 0} \tilde{a}_{j}(z, X) n^{-j / k},
$$

where the $\left\{\tilde{a}_{j}(z, X)\right\}$ are functionals. Then (2.20) means that $\exp \left(n G^{*}\right) J_{n}$ has the asymptotic expansion

$$
e^{n G^{*}} J_{n} \sim n^{1 / 2-1 / k} \sum_{j \geqslant 0}\left\{\int_{\mathbf{R} \times \mathcal{K}^{\perp}} \tilde{a}_{j}(z, X) e^{-\lambda z^{k}} d z d P_{B_{\perp}}(X)\right\} n^{-1 / k}
$$

as $n \rightarrow \infty$.

For $j$ odd, $\tilde{a}_{j}(z, X)$ turns out to be an odd function of $z$ or $X$. Since $e^{-\lambda z^{k}}$ is an even density and $P_{B_{\perp}}$ is mean zero, (2.20) has the form (1.5); i.e., only integral powers of $n^{-2 / k}$ appear.

REMARK 2.4. (a) We give the first two terms in (2.20) if $\psi \equiv 1$ and $k=4$. Even in this, the simplest simply degenerate case, the result is much more complicated than in the nondegenerate case (see Remark 2.2(a)). We find that

$$
e^{n G^{*}} J_{n}=n^{1 / 4}[2 \pi\langle A U, U\rangle \tilde{\Delta}]^{-1 / 2}\left\{1+\Gamma_{1,4} / \sqrt{n}+O\left(\frac{1}{n}\right)\right\}
$$

$$
\begin{aligned}
\Gamma_{1,4}:=\int_{\mathbf{R} \times \mathcal{K}^{\perp}}\left\{\frac{z^{2}}{8}[\right. & \left.D^{3} F\left(Y^{*}\right)\left(U, X^{2}\right)\right]^{2}-\frac{z^{2}}{2} D^{3} F\left(Y^{*}\right)\left(\Phi_{2}, X^{2}\right) \\
& -\frac{z^{2}}{4} D^{4} F\left(Y^{*}\right)\left(U^{2}, X^{2}\right) \\
& \left.+\frac{z^{10}}{2} G_{5}^{2}-z^{6} G_{6}+\frac{z^{6}}{2} G_{5} D^{3} F\left(Y^{*}\right)\left(U, X^{2}\right)\right\} e^{-\lambda z^{4}} d z d P_{B_{\perp}}(X) .
\end{aligned}
$$

The element $\Phi_{2} \in \mathcal{K}^{\perp}$ is defined by the expansion $\Phi(z U)=\Phi_{2} z^{2}+O\left(z^{3}\right)$. We find $\Phi_{2}:=-B_{\perp} D^{2} F^{\prime}\left(Y^{*}\right) U^{2}$. The numbers $\left\{G_{j}\right\}$ are defined by

$$
G\left(Y^{*}+W(z)\right)=G\left(Y^{*}\right)+G_{4} z^{4}+G_{5} z^{5}+G_{6} z^{6}+O\left(z^{7}\right) .
$$

Since $k=4$, we have $\lambda=G_{4}>0$. We find

$$
\lambda=G_{4}=D^{4} F\left(Y^{*}\right) U^{4} / 4 !-\left\langle B_{\perp} D^{2} F^{\prime}\left(Y^{*}\right) U^{2}, D^{2} F^{\prime}\left(Y^{*}\right) U^{2}\right\rangle / 8
$$

but $G_{5}$ and $G_{6}$ are in general too complicated to write out here. Our formula for $G_{4}$ also appears in [Schulman, $§ 15$, Notes], where an analogous problem involving the asymptotics of Feynman path integrals is considered.

(b) The operator $\tau \rho A \rho \tau$ in the definition of $\tilde{\Delta}$ inverts $\tau A^{-1} \tau$ on $\mathcal{K}^{\perp}$. See Lemma 5.2. 
(c) We compare the simply degenerate and the multidegenerate cases. In the former case, since $\lambda>0$ and $k \geqslant 4$ is even, we conclude that $\int z^{j} \exp \left(-\lambda z^{k}\right) d z<\infty$ for all $j \in\{0,1,2, \ldots\}$. Hence, in (2.21) we may expand around this measure in the degenerate direction. Consider now the multidegenerate case. Suppose that $d:=\operatorname{dim} \mathscr{K}>1$ and that $\left\{U_{1}, \ldots, U_{d}\right\}$ is an orthonormal basis of $\mathscr{K}$. Given $z:=\left(z_{1}, \ldots, z_{d}\right) \in \mathbf{R}^{d}$, we write $z \cdot U$ for $z_{1} U_{1}+\cdots+z_{d} U_{d}$. For all sufficiently small $z \in \mathbf{R}^{d}$, one can define $\Phi(z \cdot U) \in \mathcal{K}^{\perp}$ such that for $X \in \mathcal{K}^{\perp}$

$$
\begin{aligned}
& G\left(Y^{*}+z \cdot U+X+\Phi(z \cdot U)\right) \\
& =G\left(Y^{*}\right)+\pi_{k}(z)+\frac{1}{2}\left\langle G^{\prime \prime}\left(Y^{*}\right) X, X\right\rangle+(\text { error terms in } z, X),
\end{aligned}
$$

where $\pi_{k}(z)$ is a homogeneous polynomial of some even degree $k \geqslant 4$. The analogue of Theorem 2.3 holds if $\int_{\mathbf{R}^{d}} z^{n} \exp \left(-\pi_{k}(z)\right) d z<\infty$ for all multi-indices $n:=\left(n_{1}, \ldots, n_{d}\right)$. Instances where this integrability condition fails are readily determined. For example, in [Ellis-Newman, §6], for $\mathcal{H}:=\mathbf{R}^{2}$, a $G$ is considered for which $k=4, \pi_{4}(z)=z_{1}^{2} z_{2}^{2} / 4$. The integral of $\exp \left(-\pi_{4}(z)\right)$ over $\mathbf{R}^{2}$ is logarithmically divergent. The leading order term in the asymptotic expansion of $\exp \left(n G^{*}\right) J_{n}$ is proportional to $\log n$.

Finally, we state limit theorems for $\mathcal{G}$-valued random variables $\left\{Y_{n}\right\}$ with distributions $\left\{Q_{n}\right\}$ in (1.6). These theorems will be generalized in $\S \S I V$ and $\mathrm{V}$ to cover the case of nonunique, isolated minimum points. In these theorems, $C(\mathcal{H})$ denotes the set of all bounded, continuous, real-valued functions on $\mathcal{H}$.

THEOREM 2.5. Suppose that $G$ has a unique minimum point $Y^{*}$. Then for all functions $\phi \in C(\mathcal{H})$,

$$
\frac{\int \phi(Y / n) \exp [-n F(Y / n)] d P_{A}(Y / \sqrt{n})}{Z_{n}} \rightarrow \phi\left(Y^{*}\right)
$$

i.e., $Y_{n} / n \stackrel{\mathscr{D}}{\rightarrow} \delta_{Y^{*}}$.

This result follows from [Donsker-Varadhan, Theorem 6.2] and [Varadhan, §3]. The continuity of $F$ suffices.

THEOREM 2.6. Suppose that $G$ has a unique minimum point $Y^{*}$ and that $Y^{*}$ is nondegenerate. Then for all functions $\phi \in C(\mathcal{H})$

$$
\frac{\int \phi\left(\left(Y-n Y^{*}\right) / \sqrt{n}\right) \exp [-n F(Y / n)] d P_{A}(Y / \sqrt{n})}{Z_{n}} \rightarrow \int \phi(Y) d P_{B}(Y),
$$

where $P_{B}$ is the Gaussian measure defined in Theorem 2.1. In other words,

$$
\left(Y_{n}-n Y^{*}\right) / \sqrt{n} \stackrel{\mathscr{D}}{\rightarrow} P_{B} .
$$

Given a probability measure $\xi$ on $\mathbf{R}$ and a nonzero element $V \in \mathcal{K}, \xi^{V}$ denotes the probability measure on $\mathcal{H}$ defined by

$$
\int \phi(Y) d \xi^{V}(Y):=\int_{\mathbf{R}} \phi(z V) d \xi(z), \quad \phi \in C(\mathcal{H}) .
$$

Clearly, $\xi^{V}$ is concentrated on the span of $V$. 
THEOREM 2.7. Suppose that $G$ has a unique minimum point $Y^{*}$ and that $Y^{*}$ is simply degenerate of type $k<\infty$ and strength $\lambda$. Then for all functions $\phi \in C(\mathcal{H C})$

$$
\begin{aligned}
& \int \phi\left(\left(Y-n Y^{*}\right) / n^{1-1 / k}\right) \exp [-n F(Y / n)] d P_{A}(Y / \sqrt{n}) Z_{n} \\
& \rightarrow \frac{\int_{\mathbf{R}} \phi(z U) e^{-\lambda z^{k}} d z}{\int_{\mathbf{R}} e^{-\lambda z^{k}} d z}
\end{aligned}
$$

where $U \in \mathfrak{K},\|U\|=1$. In other words, $\left(Y_{n}-n Y^{*}\right) / n^{1-1 / k} \stackrel{\mathscr{D}}{\rightarrow} \xi_{k, \lambda}^{U}$, where $\xi_{k, \lambda}$ is the probability measure on $\mathbf{R}$ with density $\exp \left(-\lambda z^{k}\right) / \int_{\mathbf{R}} \exp \left(-\lambda z^{k}\right) d z$.

When it is interpreted in terms of random variables, the asymptotic expansion of Theorem 2.1 makes essential use of the fact that the distribution of $\left(Y_{n}-n Y^{*}\right) / \sqrt{n}$ is close to $P_{B}$. We emphasize that in distinct contrast to the nondegenerate limit (2.27), the limit $\xi_{k, \lambda}^{U}$ in the simply degenerate case is concentrated on the one-dimensional subspace $\mathscr{K}$. The nonstandard scaling $n^{1-1 / k}$ in (2.29) (where $k \geqslant 4$ ) is needed because of high correlations in the degenerate direction $U$.

III. Notation and facts needed for proofs. We denote by $\mathscr{B}(\mathcal{H C})$ the set of all bounded linear operators mapping $\mathcal{H}$ to $\mathscr{H}$. We use the same symbol $\|-\|$ to denote the norm on $\mathcal{H}$ and the derived operator norm on $\mathscr{B}(\mathcal{H})$. $F$ is assumed to be a real-valued, $C^{\infty}$-Fréchet differentiable functional on the real separable Hilbert space $\mathcal{H}$. Given $\bar{Y}, Y_{1}, Y_{2}, \ldots, \in \mathcal{H}$, we denote the $j$ th Fréchet derivative of $F$ at $\bar{Y}$ by $D F(\bar{Y}) Y_{1}$ for $j=1$ and by $D^{j} F(\bar{Y})\left(Y_{1}, \ldots, Y_{j}\right)$ for $j \geqslant 2$. If $Y_{1}=Y_{2}=\cdots=Y_{j}$, then we write $D^{j} F(\bar{Y}) Y_{1}^{j}$ instead of the latter; if for some $m \in\{1, \ldots, j-1\}, Y_{1}=\cdots=$ $Y_{m}, Y_{m+1}=\cdots=Y_{j}$, then we write $D^{j} F(\bar{Y})\left(Y_{1}^{m}, Y_{j}^{j-m}\right)$. By the Riesz representation theorem, there exist an element $F^{\prime}(\bar{Y}) \in \mathcal{H}$ and an operator $F^{\prime \prime}(\bar{Y}) \in \mathscr{B}(\mathcal{H})$ such that

$$
D F(\bar{Y}) Y_{1}=\left\langle F^{\prime}(\bar{Y}), Y_{1}\right\rangle, \quad D^{2} F(\bar{Y})\left(Y_{1}, Y_{2}\right)=\left\langle F^{\prime \prime}(\bar{Y}) Y_{1}, Y_{2}\right\rangle .
$$

Given $r>0$, we define $S(\bar{Y}, r):=\{Y: Y \in \mathcal{H},\|\bar{Y}-Y\|<r\}, \bar{S}(\bar{Y}, r):=$ $\{Y: Y \in \mathcal{H},\|\bar{Y}-Y\| \leqslant r\}$.

Let $P_{A}$ be a mean zero Gaussian measure on $\mathcal{H}$ with covariance operator $A$. In general, $A$ is a nonnegative, symmetric, trace class operator on $\mathcal{H}$ [Gihman-Skorohod, Theorem V.6.1]. As already stated, we assume (without loss of generality) that $A$ is strictly positive, $\sqrt{A}$ denotes the unique, nonnegative, symmetric square root of $A$ and $A^{-1 / 2}$ the inverse of $\sqrt{A}$. $\mathscr{D}\left(A^{-1 / 2}\right)$, the domain of $A^{-1 / 2}$, is dense in $\mathcal{H}$ and is a Hilbert space when equipped with the inner product $\left\langle Y_{1}, Y_{2}\right\rangle_{A}:=$ $\left\langle A^{-1 / 2} Y_{1}, A^{-1 / 2} Y_{2}\right\rangle$. We call this Hilbert space $\mathcal{H}_{A}$ and write its norm as $\|-\|_{A}$. Given $\bar{Y} \in \mathcal{H}_{A}, r>0$, we define

$$
\begin{aligned}
& S_{A}(\bar{Y}, r):=\left\{Y: Y \in \mathcal{H}_{A},\|\bar{Y}-Y\|_{A}<r\right\}, \\
& \overline{S_{A}}(\bar{Y}, r):=\left\{Y: Y \in \mathcal{H}_{A},\|\bar{Y}-Y\|_{A} \leqslant r\right\} .
\end{aligned}
$$

We define the entropy functional $I=I_{A}$ of $P_{A}$ by the formula

$$
I(Y):= \begin{cases}\frac{1}{2}\|Y\|_{A}^{2} & \text { if } Y \in \mathcal{H}_{A}, \\ +\infty & \text { if } Y \in \mathcal{H C}_{\mathcal{H}} \mathcal{H}_{A},\end{cases}
$$


and the functional $G$ by the formula

$$
G(Y):=F(Y)+I(Y) \text {. }
$$

If it happens that $Y \in \mathscr{D}\left(A^{-1}\right)$, then $G(Y)=F(Y)+\frac{1}{2}\left\langle A^{-1} Y, Y\right\rangle$. The functional $I$ is discussed in [Freidlin] and in [Wentzell] under the name action functional. $G$ is $C^{\infty}$-Fréchet differentiable on $\mathcal{H}_{A}$ with Fréchet derivatives

(a) $D G(\bar{Y}) Y_{1}=\left\langle F^{\prime}(\bar{Y}), Y_{1}\right\rangle+\left\langle\bar{Y}, Y_{1}\right\rangle_{A}=\left\langle A F^{\prime}(\bar{Y})+\bar{Y}, Y_{1}\right\rangle_{A}$,

(b) $D^{2} G(\bar{Y})\left(Y_{1}, Y_{2}\right)=\left\langle F^{\prime \prime}(\bar{Y}) Y_{1}, Y_{2}\right\rangle+\left\langle Y_{1}, Y_{2}\right\rangle_{A}=\left\langle A F^{\prime \prime}(\bar{Y}) Y_{1}+Y_{1}, Y_{2}\right\rangle_{A}$,

(c) $D^{j} G(\bar{Y})\left(Y_{1}, \ldots, Y_{j}\right)=D^{j} F(\bar{Y})\left(Y_{1}, \ldots, Y_{j}\right)$ if $j \geqslant 3$.

Although $G$ is not Fréchet differentiable on $\mathcal{H}$, if $\bar{Y} \in \mathcal{D}\left(A^{-1}\right)$, then we use the notation

$$
G^{\prime}(\bar{Y}):=A^{-1} \bar{Y}+F^{\prime}(\bar{Y}), \quad G^{\prime \prime}(\bar{Y}):=A^{-1}+F^{\prime \prime}(\bar{Y}) .
$$

Thus the domain of $G^{\prime \prime}(\bar{Y})$ is $\mathscr{D}\left(A^{-1}\right)$.

We define $G^{*}:=\inf \{G(Y): Y \in \mathcal{H}\}$. A point $Y^{*} \in \mathcal{H}_{A}$ is said to be a minimum point of $G$ if $G\left(Y^{*}\right)=G^{*}$. We define $\mathscr{N}$ to be the set of all minimum points of $G$. Key facts about minimum points are stated in the next lemma.

LEMma 3.1. Suppose that $F$ satisfies the bound (2.1)(b). Then minimum points of $G$ exist. If $Y^{*}$ is a minimum point, then $Y^{*} \in \mathscr{D}\left(A^{-1}\right)$,

$$
G^{\prime}\left(Y^{*}\right)=F^{\prime}\left(Y^{*}\right)+A^{-1} Y^{*}=0,
$$

and

$$
\lim _{n \rightarrow \infty} \frac{1}{n} \log \int e^{-n F(Y / \sqrt{ } n)} d P(Y)=-G\left(Y^{*}\right) .
$$

For any number $u \in\left[G^{*}, \infty\right)$ the set $\{Y: G(Y) \leqslant u\}$ is a nonempty, compact subset of $\mathcal{H}$. In particular, $\mathfrak{N}$ is compact. Hence, if the minimum points are all isolated, then they are finite in number.

Proof. If $Y^{*}$ is a minimum point then, for all $Y \in \mathcal{H}_{A}, D G\left(Y^{*}\right) Y=0$, and so by (3.4)(a) $A F^{\prime}\left(Y^{*}\right)+Y^{*}=0$. Since $F^{\prime}\left(Y^{*}\right) \in \mathcal{H}$, we see that $Y^{*} \in \mathscr{D}\left(A^{-1}\right)$ and that (3.6) holds. The limit (3.7) is proved in [Ellis-Rosen (2), Theorem A.7] as well as in [Donsker-Varadhan, Corollary 6.3]. We now prove that $\mathscr{F}:=\{Y: G(Y) \leqslant u\}$ is a nonempty, compact subset of $\mathcal{H}$ and that minimum points of $G$ exist. $G$ is lower semicontinuous (1.s.c.) since $G:=F+I$ with $F$ continuous and $I$ 1.s.c. ( $\mathcal{G}$-analogue of [Ellis-Rosen (2), Lemma 4.4(a)]). Thus, $\mathscr{F}$ is closed. For $Y \in \mathscr{F},(2.1)(\mathrm{b})$ implies $I(Y) \leqslant u+b_{4}+b_{3}\|Y\|^{2}$. Thus, for $Y \in \mathscr{F}, I(Y) \leqslant \delta^{-1}\left(u+b_{4}\right)$, where $\delta:=1-2 b_{3}\|A\|>0$. Hence $\mathscr{F}$ is compact since for any $L$ real $\{Y: I(Y) \leqslant L\}$ is compact [Ellis-Rosen, Lemma A.8]. Being 1.s.c., $G$ achieves its absolute minimum on the compact set $\mathscr{F}$. This completes the proof since inf $G$ on $\mathcal{F}$ equals inf $G$ on $\mathcal{H}$.

If $Y^{*}$ is a minimum point of $G$, then for all $Y \in \mathcal{H}_{A}, D^{2} G\left(Y^{*}\right) Y^{2} \geqslant 0$. This implies by (3.4)(b)

$$
G^{\prime \prime}\left(Y^{*}\right):=F^{\prime \prime}\left(Y^{*}\right)+A^{-1} \geqslant 0
$$


We define $\mathscr{K}:=\operatorname{ker}\left(G^{\prime \prime}\left(Y^{*}\right)\right)$. We say that $Y^{*}$ is nondegenerate, simply degenerate, or multidegenerate according to whether $\mathscr{K}=\{0\}, \operatorname{dim} \mathscr{K}=1$, or $\operatorname{dim} \mathscr{K}>1$. Since $\mathscr{K}=\left\{Y: A F^{\prime \prime}\left(Y^{*}\right) Y=-Y\right\}$, the compactness of $A F^{\prime \prime}\left(Y^{*}\right)$ implies that in general $\operatorname{dim} \mathscr{K}<\infty$.

We state a useful fact about nondegenerate minimum points.

THEOREM 3.2. If $Y^{*}$ is a nondegenerate minimum point of $G$, then $Y^{*}$ is an isolated minimum point.

The proof depends on the following lemma, which is used several times in this paper.

LEMMA 3.3. Suppose that $A_{1}$ is a symmetric, strictly positive, compact operator on a real separable Hilbert space $\mathcal{H}_{1}$ and that $\Lambda \in \mathscr{B}\left(\mathcal{H}_{1}\right)$ has the property that $A_{1}^{-1}+\Lambda>$ 0 on $\mathscr{D}\left(A_{1}^{-1}\right)$. Then there exists a constant $\nu>0$ such that for all $Y \in \mathcal{H}_{1}$

$$
\left\langle\left(I+\sqrt{A_{1}} \Lambda \sqrt{A_{1}}\right) Y, Y\right\rangle \geqslant \nu\|Y\|^{2} .
$$

Proof. Let $\left\{\mu_{j} ; j=1,2, \ldots\right\}$ be the eigenvalues of $\sqrt{A_{1}} \Lambda \sqrt{A_{1}}$. Since $\sqrt{A_{1}} \Lambda \sqrt{A_{1}}$ is compact and symmetric, it suffices to prove that $1+\mu_{j}>0$ for each $j$. If $f \in \mathcal{H}_{1}$, $f \neq 0$, satisfies $\sqrt{A_{1}} \Lambda \sqrt{A_{1}} f=\mu_{j} f$, then $f \in \mathscr{D}\left(A_{1}^{-1 / 2}\right)$ and so $g:=\sqrt{A_{1}} f \in \mathscr{D}\left(A_{1}^{-1}\right)$. Hence

$$
1+\mu_{j}=\frac{1}{\|f\|^{2}}\left\langle\left(I+\sqrt{A_{1}} \Lambda \sqrt{A_{1}}\right) f, f\right\rangle=\frac{1}{\|f\|^{2}}\left\langle\left(A_{1}^{-1}+\Lambda\right) g, g\right\rangle .
$$

Thus, $1+\mu_{j}>0$ since $A_{1}^{-1}+\Lambda>0$ on $\mathscr{D}\left(A_{1}^{-1}\right)$.

Proof of Theorem 3.2. If $Y^{*}$ is the unique minimum point of $G$, then we are done. Otherwise, since $A^{-1}$ is in general unbounded, it is easier to prove first that $Y^{*}$ is an isolated minimum point in $\mathcal{H}_{A}$, then that $Y^{*}$ is an isolated minimum point in $\mathcal{H}$. We prove the assertion about $\mathcal{H}_{A}$. By Lemma 3.3 with $\mathcal{H}_{1}:=\mathscr{H}, A_{1}:=A$, $\Lambda:=F^{\prime \prime}\left(Y^{*}\right)$, there exists $\nu>0$ such that for all $V \in \mathcal{H}_{A}$

$$
\left\langle\left(I+\sqrt{A} F^{\prime \prime}\left(Y^{*}\right) \sqrt{A}\right) A^{-1 / 2} V, A^{-1 / 2} V\right\rangle \geqslant \nu\left\|A^{-1 / 2} V\right\|^{2} .
$$

By the smoothness of $F$, there exists $\nu>0$ such that for all $Y \in \bar{S}_{A}\left(Y^{*}, r\right)$

$$
\left\langle\left(I+\sqrt{A} F^{\prime \prime}(Y) \sqrt{A}\right) A^{-1 / 2} V, A^{-1 / 2} V\right\rangle \geqslant \frac{\nu}{2}\left\|A^{-1 / 2} V\right\|^{2} .
$$

But (3.12) shows that for all $V \in \mathcal{H}_{A}$

$$
D^{2} G(Y) V^{2}=\left\langle\left(I+A F^{\prime \prime}(Y)\right) V, V\right\rangle_{A} \geqslant \frac{\nu}{2}\|V\|_{A}^{2} .
$$

Hence by [Berger, Theorem 3.2.2] there exists a number $r_{1} \in(0, r]$ such that $Y^{*}$ is the unique minimum point of $G$ in $\bar{S}_{A}\left(Y^{*}, r_{1}\right)$.

We now prove the assertion about $\mathcal{H}$. Assume, on the contrary, that $Y^{*}$ is not an isolated minimum point in $\mathcal{H}$. Then we may find a sequence $\left\{Y_{m}^{*}\right\}$ of minimum points such that $\left\|Y^{*}-Y_{m}^{*}\right\| \rightarrow 0$ as $m \rightarrow \infty$. By the smoothness of $F$, this would imply $\left\|F^{\prime}\left(Y^{*}\right)-F^{\prime}\left(Y_{m}^{*}\right)\right\| \rightarrow 0$ and thus by (3.6), $\left\|A^{-1} Y^{*}-A^{-1} Y_{m}^{*}\right\| \rightarrow 0$. But then we would have $\left\|Y^{*}-Y_{m}^{*}\right\|_{A}:=\left\langle A^{-1} Y^{*}-A^{-1} Y_{m}, Y^{*}-Y_{m}^{*}\right\rangle \rightarrow 0$, which contradicts the first part of this proof. 
IV. Proofs of Theorems 2.1, 2.6, and extensions (nondegenerate case). The plan of this section is to prove Theorems 2.1 and 2.6 together with extensions of Theorems 2.5 and 2.6. The extensions cover the case where $G$ has nonunique minimum points all of which are nondegenerate. By Lemma 3.1 and Theorem 3.2, these minimum points must be finite in number. These extensions are stated and proved as Theorem 4.6 at the end of the section.

The proof of Theorem 2.1 depends on three lemmas, Lemmas 4.1-4.3, which are stated just below. After stating the lemmas, we prove Theorem 2.1, then prove the lemmas. Lemma 4.1 shows that the main contribution to $\exp \left(n G^{*}\right) J_{n}$ comes from a neighborhood of $Y^{*}$. Lemma 4.2 is the heart of the proof. It expresses this contribution in a usable form that allows one to complete the analysis. Lemma 4.3 gives an important bound on certain tail probabilities. We state Lemmas 4.1-4.2 in greater generality than is needed to prove Theorem 2.1 since they will also be used to prove Theorem 4.6, in which it is assumed that $G$ has nonunique minimum points.

LEMMA 4.1. Let $\Re$ denote the set of minimum points of $G$. Suppose that $\psi$ and $F$ satisfy the bounds (2.1)(a)-(b). Then for any $\delta>0$ there exists $c=c(\delta)>0$ such that

$$
e^{n G^{*}} \int_{\left\{Y:\left\|Y / \sqrt{ }-Y^{*}\right\|>\delta, \text { all } Y^{*} \in \mathscr{N}\right\}} \psi(Y / \sqrt{n}) e^{-n F(Y / \sqrt{ } n)} d P_{A}(Y)=O\left(e^{-n c}\right)
$$

REMARK 4.1(a). The only smoothness required of $\psi$ in (4.1) is that it be continuous. This form of Lemma 4.1 is used below in the proofs of Theorems 2.6, 2.7, 4.6, and 5.5.

LEMMA 4.2. Suppose that $Y^{*}$ is a nondegenerate minimum point of $G$. Then

$$
\Delta=\Delta\left(Y^{*}\right):=\operatorname{det}\left(I+A F^{\prime \prime}\left(Y^{*}\right)\right)
$$

is well defined and $\Delta>0$. Also, $G^{\prime \prime}\left(Y^{*}\right)$ is invertible on $\mathcal{H}$ and

$$
B=B\left(Y^{*}\right):=\left(G^{\prime \prime}\left(Y^{*}\right)\right)^{-1}
$$

is the covariance operator of a mean zero Gaussian measure $P_{B}$ on $\mathcal{H}$. Define the functional $F_{3}=F_{3}\left(Y^{*} ; \cdot\right)$ by (2.3). Then for any $\delta>0$

$$
\begin{aligned}
e^{n G^{*}} \int_{S\left(\sqrt{ } n Y^{*}, \sqrt{ } n \delta\right)} \psi & (Y / \sqrt{n}) e^{-n F(Y / \sqrt{ } n)} d P_{A}(Y) \\
& =\Delta^{-1 / 2} \int_{S(0, \sqrt{ } \delta)} \psi\left(Y^{*}+Y / \sqrt{n}\right) e^{-n F_{3}(Y / \sqrt{ } n)} d P_{B}(Y)
\end{aligned}
$$

REMARK 4.2(a). The only smoothness required of $\psi$ in (4.4) is that it be continuous. This form of Lemma 4.2 is used below in the proofs of Theorems 2.6, 4.6, and 5.5 .

LemMa 4.3. Let $P$ be any mean zero Gaussian measure on $\mathcal{H}$ and let $A_{1}$ be its covariance operator. For any $b \in\left(0,1 /\left(2\left\|A_{1}\right\|\right)\right)$, we have $\int \exp \left(b\|Y\|^{2}\right) d P(Y)<\infty$. For any such $b$ there exists $c=c(b)>0$ such that

$$
P\{Y:\|Y\|>a\} \leqslant c e^{-b a^{2}} \text { for all } a \geqslant 0 .
$$


Proof of Theorem 2.1. The facts about $\Delta, B, P_{B}$ are proved in Lemma 4.2. We prove (2.4) by showing that there exist functionals $\left\{\Gamma_{j} ; j=0,1, \ldots\right\}$ such that for any integer $M \geqslant 0$

$$
e^{n G^{*}} J_{n}=\sum_{j=0}^{M} \Gamma_{j} n^{-j}+O\left(n^{-(M+1)}\right) \quad \text { as } n \rightarrow \infty .
$$

The $\left\{\Gamma_{j}\right\}$ are found by expanding the integrand in (2.4) in powers of $Y / \sqrt{n}$ and then by integrating term-by-term with respect to $d P_{B}$ over $\mathcal{H}$. Since $P_{B}$ is mean zero, only integral powers of $n^{-1}$ survive.

Since $\Re=\left\{Y^{*}\right\}$, the domain of integration in (4.1) is $\left[S\left(\sqrt{n} Y^{*}, \sqrt{n} \delta\right)\right]^{c}$. Hence by Lemmas 4.1-4.2, for any $\delta>0$

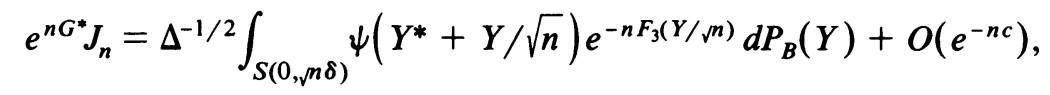

where $c=c(\delta)>0$. We show how to go from (4.7) to (2.4). Let an integer $M \geqslant 0$ be given. Pick $\delta$ so small that $\left\|D^{2 m+2} \psi\left(Y^{*}+Y\right)\right\|,\left\|D^{3} F\left(Y^{*}+Y\right)\right\|$, $\left\|D^{2 M+4} F\left(Y^{*}+Y\right)\right\|$ are uniformly bounded on $S(0, \delta)$. By Taylor's theorem [Berger, Theorem 2.1.33], we have for all $Y \in S(0, \sqrt{n} \delta)$

$$
\psi\left(Y^{*}+Y / \sqrt{n}\right)=\sum_{j=0}^{2 M+1} \frac{1}{j !} D^{j} \psi\left(Y^{*}\right)\left(\frac{Y}{\sqrt{n}}\right)^{j}+O\left(\|Y\|^{2 M+2} n^{-(M+1)}\right)
$$

and

$$
-n F_{3}(Y / \sqrt{n})=\sum_{j=3}^{2 M+3} \frac{-1}{j !} D^{j} F\left(Y^{*}\right) \frac{Y^{j}}{n^{j / 2-1}}+O\left(\|Y\|^{2 M+4} n^{-(M+1)}\right) .
$$

We also note that if $\|Y / \sqrt{n}\|<\delta$, then

$$
\left|n F_{3}(Y / \sqrt{n})\right|=O\left(\|Y\|^{3} / \sqrt{n}\right)=O\left(\delta\|Y\|^{2}\right) .
$$

(This shows that the term $n F_{3}(Y / \sqrt{n})$ in (2.4) is an error term.)

For any real number $x$ we have

$$
e^{x}=\sum_{i=0}^{2 M+1} x^{i} / i !+S_{M}, \quad \text { where }\left|S_{M}\right| \leqslant e^{|x|}|x|^{2 M+2} .
$$

Hence for all $Y \in S(0, \sqrt{n} \delta)$, we have

$$
\begin{gathered}
\exp \left[-n F_{3}(Y / \sqrt{n})\right]=1+\sum_{i=1}^{2 M+1}\left(-n F_{3}(Y / \sqrt{n})\right)^{i} / i !+S_{M} \\
\left|S_{M}\right| \leqslant \exp \left[\left|n F_{3}(Y / \sqrt{n})\right|\right]\left|n F_{3}(Y / \sqrt{n})\right|^{2 M+2} \\
=O\left(\exp \left[c_{1} \delta\|Y\|^{2}\right]\|Y\|^{6(M+1)} n^{-(M+1)}\right)
\end{gathered}
$$

where $c_{1}=c_{1}(\delta)>0$. The last estimate uses (4.10). We substitute (4.9) into the right-hand side of (4.12), then substitute (4.8), (4.12) into (4.7). There exists an 
integer $N=N(M)$ and for each $i \in\{0, \ldots, 2 M+1\}$ there exist functionals $\Xi_{i}(Y)$ such that

$$
e^{n G\left(Y^{*}\right)} J_{n}=\Delta^{-1 / 2} \sum_{i=0}^{2 M+1} n^{-i / 2} \int_{S(0, n n \delta)} \Xi_{i}(Y) d P_{B}(Y)+T_{M}(n)
$$

where

$$
\left|T_{M}(n)\right| \leqslant \text { const }\left[e^{-n c}+\frac{1}{n^{(M+1)}} \int_{S(0, n n \delta)}\left[1+\|Y\|^{N(M)}\right] e^{c_{1} \delta\|Y\|^{2}} d P_{B}(Y)\right]
$$

By reducing $\delta$, if necessary, we conclude by Lemma 4.3 that

$$
T_{M}(n)=O\left(n^{-(M+1)}\right) .
$$

In (4.14), $\Xi_{0}=\psi\left(Y^{*}\right)$ and, for $i \in\{1,2, \ldots, 2 M+1\}, \Xi_{i}$ is a sum of products involving $D^{j} \psi\left(Y^{*}\right) Y^{j}$ and $D^{l} F\left(Y^{*}\right) Y^{l}$ for $j \in\{0, \ldots, 2 M+1\}$ and $l \in$ $\{3, \ldots, 2 M+3\}$. If $i$ is odd, then $\Xi_{i}$ is an odd function of $Y$.

We claim that for each $i \in\{0, \ldots, 2 M+1\}$

$$
\int_{S(0, n n \delta)} \Xi_{i}(Y) d P_{B}(Y)=\int \Xi_{i}(Y) d P_{B}(Y)+O\left(e^{-n c_{2}}\right)
$$

for some $c_{2}>0$. Since $P_{B}$ is mean zero and $\Xi_{i}$ is an odd function of $Y$ for odd $i$, $\int \Xi_{i} d P_{B}=0$ for odd $i$. Substituting (4.16)-(4.17) into (4.14), we obtain the expansion (4.6) with

$$
\Gamma_{j}:=\Delta^{-1 / 2} \int \Xi_{2 j}(Y) d P_{B}(Y) .
$$

We prove (4.17). For each $i \in\{0, \ldots, 2 M+1\}$, there exists an integer $N_{i} \geqslant 0$ such that $\left|\Xi_{i}(Y)\right| \leqslant \operatorname{const}\left(1+\|Y\|^{N_{i}}\right)$. Hence (4.17) follows from Lemma 4.3.

We next prove Lemmas 4.1, 4.3 and 4.2.

Proof of Lemma 4.1. Let $\mathcal{L}$ be any closed subset of $\mathcal{H}$ such that $\mathcal{L} \cap \mathfrak{N}=\varnothing$. We first prove that there exists $D>0$ such that

$$
\inf \{G(Y): Y \in \mathcal{L}\}-G^{*}>D \text {. }
$$

Suppose that (4.19) is false. Then there exists a sequence $\left\{\bar{Y}_{m} ; m=1,2, \ldots\right\}$ in $\mathcal{L}$ such that $G\left(\bar{Y}_{m}\right) \rightarrow G^{*}$ and $G\left(\bar{Y}_{m}\right) \leqslant G^{*}+1$ for each $\Re$. By Lemma 3.1, the set $\left\{\bar{Y}_{m}\right\}$ is conditionally compact and so there exists an element $\bar{Y}$ such that $\left\|\bar{Y}-\bar{Y}_{m}\right\|$ $\rightarrow 0$. Since $\mathcal{E}$ is closed, we have $\bar{Y} \in \mathcal{L}$. By [Ellis-Rosen (2), Lemma 4.5(a)] (with $\mathcal{H}$ written for $C[0,1]), G$ is lower semicontinuous, and so

$$
G^{*}=\lim _{m \rightarrow \infty} G\left(\bar{Y}_{m}\right) \geqslant G(\bar{Y}) .
$$

Since we must have $G(\bar{Y}) \geqslant G^{*}$, we conclude $G(\bar{Y})=G^{*}$ or $\bar{Y} \in \mathfrak{T}$. Thus $\bar{Y} \in \mathcal{L}$ $\cap \mathfrak{T}$, which is impossible. This contradiction proves (4.19). By [Donsker-Varadhan, Theorem 6.2] and [Varadhan, §3], (4.19) implies

$$
Q_{n}\{Y: Y / n \in \mathcal{L}\}=O\left(e^{-n D}\right),
$$

where $Q_{n}$ is the measure defined in (1.6). The limit (3.7) implies that for any $\varepsilon>0$

$$
e^{n G^{*}}=O\left(e^{n \varepsilon} / Z(n)\right) \text {, }
$$


where $Z(n)$ is defined in (1.7). The bounds (4.20)-(4.21) imply that

$$
e^{n G^{*}} \int_{\mathcal{L}} e^{-n F(Y)} d P_{A}(\sqrt{n} Y)=O\left(e^{-n(D-\varepsilon)}\right) .
$$

We pick $\varepsilon \in(0, D)$. (If $|\psi|$ were uniformly bounded on $\mathcal{H}$, then (4.22) would imply (4.1).) By Lemma 3.1, $\Re$ is compact and so there exists $\bar{R}>0$ such that $\Re \subset$ $\{Y:\|Y\| \leqslant \bar{R} / 2\}$. For fixed $\delta>0$ and any sufficiently large $R$ the set

$$
\mathfrak{L}_{R}:=\{Y:\|Y-\bar{Y}\| \geqslant \delta \text {, all } \bar{Y} \in \mathfrak{R} ;\|Y\| \leqslant R\}
$$

is nonempty and closed. For any such $R$, the left-hand side of (4.1) is bounded by

$$
\begin{aligned}
e^{n G^{*}} \int_{\mathfrak{L}_{R}}|\psi(Y)| e^{-n F(Y)} d P_{A}(\sqrt{n} Y) & \\
& +e^{n G^{*}} \int_{\{Y:\|Y\|>R\}}|\psi(Y)| e^{-n F(Y)} d P_{A}(\sqrt{n} Y) .
\end{aligned}
$$

Below we shall choose a suitable value of $R$. Since $|\psi|$ is uniformly bounded on $\mathcal{L}_{R}$, (4.22) implies that the first term in (4.24) is bounded by $O(\exp (-n(D-\varepsilon)))$. By (2.1)(b) the second term in (4.24) is bounded by

$$
b_{1} \exp \left[n\left(G\left(Y^{*}\right)+b_{4}\right)\right] \int_{\{Y:\|Y\|>R\}} \exp \left[n\left(b_{3}+\frac{b_{2}}{n}\right)\|Y\|^{2}\right] d P_{A}(\sqrt{n} Y) .
$$

Since $b_{3} \in(0,1 /(2\|A\|))$, we can pick $q>1$ and $\varepsilon_{1}>0$ such that $q\left(b_{3}+b_{2} / n\right)<$ $1 /(2\|A\|)-\varepsilon_{1}$ for all $n$ sufficiently large. Let $p$ be the conjugate exponent to $q$. Then by Lemma $4.3,(4.25)$ is bounded by

$$
\begin{aligned}
b_{1} \exp [ & \left.n\left(G^{*}+b_{4}\right)\right]\left[P_{A}\{Y:\|Y\|>R \sqrt{n}\}\right]^{1 / p} \\
\cdot & {\left[\int \exp \left[\left(\frac{1}{2\|A\|}-\varepsilon_{1}\right)\|Y\|^{2}\right] d P_{A}(Y)\right]^{1 / q}=O\left(\exp \left[n\left(G^{*}+b_{4}-b R^{2} / p\right)\right]\right) }
\end{aligned}
$$

for some $b>0$. This is bounded by $O(\exp (-n(D-\varepsilon)))$ provided we pick a sufficiently large $R$. Thus, both terms in (4.24) have been suitably bounded and we obtain (4.1).

Proof of LEMma 4.3. The first assertion is proved in [Gihman-Skorohod, p. 351]. The second assertion follows from the first via Chebyshev's inequality.

Proof of Lemma 4.2. We shall derive the facts about $\Delta$ and $B$ from the following lemma. This lemma, which will be proved after the proof of Lemma 4.2, is also used later in this paper.

LEMMA 4.4. Suppose that $A_{1}$ is a symmetric, strictly positive, trace class operator on a real separable Hilbert space $\mathcal{H}_{1}$ and that $\Lambda$ is a symmetric operator in $\mathscr{B}_{(}\left(\mathcal{H}_{1}\right)$ such that $A_{1}^{-1}+\Lambda>0$ on $\mathscr{D}\left(A_{1}^{-1}\right)$. Then

$$
\Delta_{1}:=\operatorname{det}\left(I+A_{1} \Lambda\right)=\operatorname{det}\left(I+\sqrt{A_{1}} \Lambda \sqrt{A_{1}}\right)
$$

is well defined and $\Delta_{1}>0$. Also, $B_{1}:=\left(A_{1}^{-1}+\Lambda\right)^{-1}$ exists and is given by

$$
B_{1}=\sqrt{A_{1}}\left(I+\sqrt{A_{1}} \Lambda \sqrt{A_{1}}\right)^{-1} \sqrt{A_{1}} \text {. }
$$


$B_{1}$ is the covariance operator of a mean zero Gaussian measure $P_{B_{1}}$ on $\mathcal{H}_{1}$ and

$$
d P_{B_{1}}(Y)=\sqrt{\Delta_{1}} \exp \left(-\frac{1}{2}\langle\Lambda Y, Y\rangle\right) d P_{A_{1}}(Y) .
$$

The facts about $\Delta$ and $B$ follow from Lemma 4.4. Indeed, by definition of nondegeneracy, the hypotheses of this lemma are satisfied with $\mathcal{H}_{1}:=\mathcal{H}, A_{1}:=A$, and $\Lambda:=F^{\prime \prime}\left(Y^{*}\right)$. In addition, (4.28) implies that for $Y \in \mathscr{H}$

$$
d P_{B}(Y)=\sqrt{\Delta} \exp \left(-\frac{1}{2}\left\langle F^{\prime \prime}\left(Y^{*}\right) Y, Y\right\rangle\right) d P_{A}(Y) .
$$

We need one more fact in order to prove (4.4). This fact follows from [GihmanSkorohod, Theorem VII.4.1].

LEMMA 4.5. Let $P$ be any mean zero Gaussian measure on $\mathcal{H}, A_{1}$ its covariance operator, and $I_{A_{1}}$ its entropy functional (see (3.2)). If $V \in \mathscr{D}\left(A_{1}^{-1}\right)$, then $P(\cdot+V) \ll P$ and

$$
(d P(\cdot+V) / d P)(Y)=\exp \left[-I_{A_{1}}(V)-\left\langle A_{1}^{-1} V, Y\right\rangle\right]
$$

We are now ready to prove (4.4). In the integral on the left-hand side of (4.4), we translate $Y \rightarrow Y+\sqrt{n} Y^{*}$. By Lemma 3.1, $Y^{*} \in \mathcal{D}\left(A^{-1}\right)$ so that by Lemma 4.5 , the left-hand side of (4.4) equals

$$
\begin{aligned}
e^{n G\left(Y^{*}\right)} \int_{S(0, \sqrt[n]{n} \delta)} \psi\left(Y^{*}+Y / \sqrt{n}\right) \\
\quad \cdot \exp \left[-n\left\{F\left(Y^{*}+Y / \sqrt{n}\right)+I\left(Y^{*}\right)+\left\langle A^{-1} Y^{*}, Y / \sqrt{n}\right\rangle\right\}\right] d P_{A}(Y) .
\end{aligned}
$$

In (4.30), we substitute

$F\left(Y^{*}+Y / \sqrt{n}\right)=F\left(Y^{*}\right)+\left\langle F^{\prime}\left(Y^{*}\right), Y / \sqrt{n}\right\rangle+\frac{1}{2 n}\left\langle F^{\prime \prime}\left(Y^{*}\right) Y, Y\right\rangle+F_{3}\left(Y^{*} ; Y / \sqrt{n}\right)$.

By (3.3) and (3.6), (4.30) equals

$$
\int_{S(0, n n \delta)} \psi\left(Y^{*}+Y / \sqrt{n}\right) \exp \left[-n F_{3}\left(Y^{*} ; Y / \sqrt{n}\right)\right] \exp \left[-\frac{1}{2}\left\langle F^{\prime \prime}\left(Y^{*}\right) Y, Y\right\rangle\right] d P_{A}(Y)
$$

This equals the right-hand side of (4.4) by (4.29).

Proof OF LEMMA 4.4. In order to prove the statements about $\Delta_{1}$, we use [Gohberg-Kreĭn; Chapter IV.1]. $A_{1}$, and thus $A_{1} \Lambda$, are trace class operators on $\mathcal{H}_{1}$. Thus, $\Delta_{1}$ is well defined. We now prove $\Delta_{1}>0$. Writing $\left\{\mu_{j} ; j=1,2, \ldots\right\}$ for the eigenvalues of $\sqrt{A_{1}} \Lambda \sqrt{A_{1}}$, we have

$$
\Delta_{1}=\operatorname{det}\left(I+\sqrt{A_{1}} \Lambda \sqrt{A_{1}}\right)=\prod_{j=1}^{\infty}\left(1+\mu_{j}\right) .
$$

Hence it suffices to prove that $1+\mu_{j}>0$ for each $j$. This follows from Lemma 3.3.

We next prove the statements about $B_{1}$. On $\mathscr{D}\left(A_{1}^{-1}\right)$, we have

$$
A_{1}^{-1}+\Lambda=A_{1}^{-1 / 2}\left(I+\sqrt{A_{1}} \Lambda \sqrt{A_{1}}\right) A_{1}^{-1 / 2} \text {. }
$$


By Lemma 3.3, there exists a constant $\nu>0$ such that $\left(I+\sqrt{A_{1}} \Lambda_{\sqrt{A_{1}}}\right) \geqslant \nu$. There also exists a constant $\bar{\nu}>\nu$ such that $\bar{\nu} \geqslant\left(I+\sqrt{A_{1}} \Lambda \sqrt{A_{1}}\right)$. By [Riesz-Sz.-Nagy, pp. 265-266], $\left(I+\sqrt{A_{1}} \Lambda \sqrt{A_{1}}\right)$ is invertible. Hence $\left(A_{1}^{-1}+\Lambda\right)$ is invertible and we have (4.27). Since (4.27) exhibits $B_{1}$ as a nonnegative, symmetric, trace class operator, $B_{1}$ is the covariance operator of a mean zero Gaussian measure $P_{B_{1}}$ on $\mathcal{H}$ [GihmanSkorohod, Theorem V.6.7]. Equation (4.28) follows from [Gihman-Skorohod, Theorem VII.4.4 and Remark, p. 498] (with their $V:=A_{1} \Lambda$ ).

We now turn to the probabilistic limit theorems. We first prove Theorem 2.6 , then state and prove extensions of Theorems 2.5-2.6 to the case where $G$ has nonunique minimum points all of which are degenerate. These extensions are given in Theorem 4.6. We prove Theorems 2.6 and 4.6 by applying Lemmas 4.1 and 4.2 as modified by Remarks 4.1(a) and 4.2(a) respectively.

Proof of Theorem 2.6. We prove that for any $\phi \in C(\mathcal{H})$

$$
\frac{\int \phi\left(Y-\sqrt{n} Y^{*}\right) \exp [-n F(Y / \sqrt{n})] d P_{A}(Y)}{\int \exp [-n F(Y / \sqrt{n})] d P_{A}(Y)} \rightarrow \int \phi(Y) d P_{B}(Y) .
$$

We multiply the numerator and denominator of the left-hand side of (4.35) by $e^{n G^{*}}$. By Lemma 4.1, the resulting quotient equals

$$
\frac{e^{n G^{*}} \int_{S\left(\sqrt{ } n Y^{*}, \sqrt{ } n \delta\right)} \phi\left(Y-\sqrt{n} Y^{*}\right) \exp [-n F(Y / \sqrt{n})] d P_{A}(Y)+O\left(e^{-n c}\right)}{e^{n G^{*}} \int_{S\left(\sqrt{ } n Y^{*}, \sqrt{ } n \delta\right)} \exp [-n F(Y / \sqrt{n})] d P_{A}(Y)+O\left(e^{-n c}\right)}
$$

for any $\delta>0$ and $c=c(\delta)>0$. We have by Lemma 4.2 that (4.36) equals

$$
\frac{\Delta^{-1 / 2} \int_{S(0, \sqrt{ } n \delta)} \phi(Y) e^{-n F_{3}(Y / \sqrt{ } n)} d P_{B}(Y)+O\left(e^{-n c}\right)}{\Delta^{-1 / 2} \int_{S(0, \sqrt{ } n \delta)} e^{-n F_{3}(Y / \sqrt{ } n)} d P_{B}(Y)+O\left(e^{-n c}\right)} .
$$

We want to use the dominated convergence theorem to study the $n \rightarrow \infty$ limits of the integrals in (4.37). Since

$$
\left|n F_{3}(Y / \sqrt{n})\right| \leqslant \int_{0}^{1}(1-s)\left|\left[D^{2} F\left(Y^{*}+\frac{s Y}{\sqrt{n}}\right)-D^{2} F\left(Y^{*}\right)\right] Y^{2}\right| d s,
$$

the smoothness of $F$ implies that given $b>0$ there exists $\delta_{1} \in(0, \delta]$ such that if $Y$ satisfies $\|Y / \sqrt{n}\|<\delta_{1}$ then, for any $n,\left|n F_{3}(Y / \sqrt{n})\right| \leqslant b\|Y\|^{2}$. We replace $\delta$ in (4.36)-(4.37) by $\delta_{1}$. Lemma 4.3 shows that the dominated convergence theorem is applicable provided $b$ and thus $\delta_{1}$ are sufficiently small. For any fixed $Y,(4.38)$ implies that $n F_{3}(Y / \sqrt{n}) \rightarrow 0$ as $n \rightarrow \infty$; also, $\chi_{S(0, n \delta)}(Y) \rightarrow 1$. We conclude that as $n \rightarrow \infty$ the quotient in (4.37) equals

$$
\frac{\Delta^{-1 / 2} \int \phi(Y) d P_{B}(Y)+o(1)+O\left(e^{-n c}\right)}{\Delta^{-1 / 2} \int d P_{B}(Y)+o(1)+O\left(e^{-n c}\right)}=\int \phi(Y) d P_{B}(Y)+o(1)
$$

provided $\delta=\delta_{1}$ is sufficiently small. This gives (4.35).

We now assume that $G$ has nonunique minimum points $\left\{Y_{\alpha}^{*} ; \alpha=1, \ldots, L\right\}$ all of which are nondegenerate. Thus we have $L \in\{2,3, \ldots\}$. We extend Theorems 
2.5-2.6 to cover this case. The following quantities arise in these extensions. For each $\alpha \in\{1, \ldots, L\}$, we define

$$
\bar{b}_{\alpha}:=\left[\operatorname{det}\left(I+A F^{\prime \prime}\left(Y_{\alpha}^{*}\right)\right)\right]^{-1 / 2}, \quad b_{\alpha}:=\bar{b}_{\alpha} / \sum_{\beta=1}^{L} \bar{b}_{\beta},
$$

and $B\left(Y_{\alpha}^{*}\right):=\left[G^{\prime \prime}\left(Y_{\alpha}^{*}\right)\right]^{-1}$. By Lemma 4.2, the $\left\{\bar{b}_{\alpha}\right\}$ are well-defined, each $\bar{b}_{\alpha}>0$, and each $B\left(Y_{\alpha}^{*}\right)$ exists and is the covariance operator of a mean zero Gaussian measure $P_{B\left(Y_{\alpha}^{*}\right)}$ on $\mathcal{H}$.

A second type of convergence arises in the extensions of Theorems 2.5-2.6. We say that a function $\phi: \mathcal{K} \rightarrow \mathbf{R}$ vanishes at infinity if $\phi\left(Y_{m}\right) \rightarrow 0$ for any sequence $\left\{Y_{m}\right\}$ such that $\left\|Y_{m}\right\| \rightarrow \infty$. Given probability measures $\left\{\xi_{n} ; n=1,2, \ldots\right\}$ and $\xi$ on $\mathcal{H}$, we say that the $\left\{\xi_{n}\right\}$ tend vaguely to $\xi$, and write $\xi_{n} \stackrel{v}{\rightarrow} \xi$, if $\int \phi d \xi_{n} \rightarrow \int \phi d \xi$ for all $\phi \in C(\mathcal{H})$ which vanish at infinity. If $\left\{X_{n}\right\}$ are $\mathcal{K}$-valued random variables with distributions $\left\{\xi_{n}\right\}$, we also write $X_{n} \stackrel{\bullet}{\rightarrow} \xi$.

In the next theorem, (4.41) generalizes Theorem 2.5 and (4.42) generalizes Theorem 2.6. We recall that the $\left\{Y_{n}\right\}$ are $\mathcal{F}$-valued random variables with distributions $\left\{Q_{n}\right\}$ in (1.6).

THeOREM 4.6. Suppose that $G$ has minimum points $\left\{Y_{\alpha}^{*} ; \alpha=1, \ldots, L\right\}$ and that each $Y^{*}$ is nondegenerate. Suppose that $F$ satisfies the bound $(2.1)(\mathrm{b})$. Then for all $\phi \in C(\mathcal{H})$

$$
\frac{\int \phi(Y / n) \exp (-n F(Y / n)) d P_{A}(Y / \sqrt{n})}{Z_{n}} \rightarrow \sum_{\alpha=1}^{L} b_{a} \phi\left(Y_{\alpha}^{*}\right)
$$

i.e., $Y_{n} / n \stackrel{\mathcal{D}}{\rightarrow} \sum_{\alpha=1}^{L} b_{\alpha} \delta_{Y_{\alpha}^{*}}$. For each $\alpha \in\{1, \ldots, L\}$ and for all $\phi \in C(\mathcal{H C})$ which vanish at infinity,

$$
\frac{\int \phi\left(\left(Y-n Y_{\alpha}^{*}\right) / \sqrt{n}\right) \exp (-n F(Y / n)) d P_{A}(Y / \sqrt{n})}{Z_{n}} \rightarrow b_{\alpha} \int \phi(Y) d P_{B\left(Y_{\alpha}^{*}\right)}(Y) ;
$$

i.e., $\left(Y_{n}-n Y_{\alpha}^{*}\right) / \sqrt{n} \stackrel{v}{\rightarrow} b_{\alpha} P_{B\left(Y_{\alpha}^{*}\right)}$.

Proof. To prove (4.41), we prove that for any $\phi \in C(\mathcal{H C})$

$$
\frac{\int \phi(Y / \sqrt{n}) \exp [-n F(Y / \sqrt{n})] d P_{A}(Y)}{\int \exp [-n F(Y / \sqrt{n})] d P_{A}(Y)} \rightarrow \sum_{\alpha=1}^{L} b_{\alpha} \phi\left(Y_{\alpha}^{*}\right)
$$

as $n \rightarrow \infty$. For $\delta>0$ and $\alpha \in\{1, \ldots, L\}$, we write $\Sigma(\alpha, \delta)$ for $S\left(\sqrt{n} Y_{\alpha}^{*}, \sqrt{n} \delta\right)$. We multiply the numerator and denominator of the middle term in (4.43) by $\exp \left(n G^{*}\right)$. By Lemma 4.1 the resulting quotient equals

$$
\frac{\sum_{\alpha=1}^{L} e^{n G^{*}} \int_{\Sigma(\alpha, \delta)} \phi(Y / \sqrt{n}) \exp [-n F(Y / \sqrt{n})] d P_{A}(Y)+O\left(e^{-n c}\right)}{\sum_{\alpha=1}^{L} e^{n G^{*}} \int_{\Sigma(\alpha, \delta)} \exp [-n F(Y / \sqrt{n})] d P_{A}(Y)+O\left(e^{-n c}\right)}
$$


for any $\delta>0$ so small that $\Sigma(\alpha, \delta) \cap \Sigma(\beta, \delta)=\varnothing$ if $\alpha \neq \beta$ and $c=c(\delta)>0$. We apply Lemma 4.2 to each summand in (4.44). We have for each $\alpha \in\{1, \ldots, L\}$

$$
\begin{aligned}
e^{n G^{*}} \int_{\Sigma(\alpha, \delta)} & \tilde{\phi}(Y / \sqrt{n}) \exp [-n F(Y / \sqrt{n})] d P_{A}(Y) \\
& =\bar{b}_{\alpha} \int_{S(0, \sqrt{ } \delta \delta)} \tilde{\phi}\left(Y_{\alpha}^{*}+Y / \sqrt{n}\right) e^{-n F_{3}\left(Y_{\alpha}^{*} ; Y / \sqrt{ } n\right)} d P_{B\left(Y_{\alpha}^{*}\right)},
\end{aligned}
$$

where $\tilde{\phi}$ equals $\phi$ or 1 . As in the proof of Theorem 2.6, provided $\delta$ is sufficiently small, we may apply the dominated convergence theorem to conclude that as $n \rightarrow \infty$, the quotient in (4.44) equals

$$
\frac{\sum_{\alpha=1}^{L} \bar{b}_{\alpha}\left[\phi\left(Y_{\alpha}^{*}\right)+o(1)\right]+O\left(e^{-n c}\right)}{\sum_{\alpha=1}^{L} \bar{b}_{\alpha}[1+o(1)]+O\left(e^{-n c}\right)} .
$$

This gives (4.43) and thus (4.41).

We prove (4.42) for $\alpha=1$. Define $d T_{\alpha}(Y):=e^{-n F_{3}\left(Y_{\alpha}^{*} ; Y / \sqrt{n}\right)} d P_{B\left(Y_{\alpha}^{*}\right)}(Y)$. Proceeding as in the proof of (4.41), we have that the left-hand side of (4.42) equals

$$
\begin{aligned}
& {\left[\sum_{\alpha=1}^{L} \bar{b}_{\alpha} \int_{S(0, \sqrt{ } n \delta)} d T_{\alpha}(Y)+O\left(e^{-n c}\right)\right]^{-1} } \\
& \cdot\left[\bar{b}_{1} \int_{S(0, \sqrt{ } n \delta)} \phi(Y) d T_{1}(Y)\right. \\
&\left.+\sum_{\alpha=2}^{L} \bar{b}_{\alpha} \int_{S(0, \sqrt{ } n \delta)} \phi\left(Y+\sqrt{n}\left(Y_{\alpha}^{*}-Y_{1}^{*}\right)\right) d T_{\alpha}(Y)+O\left(e^{-n c}\right)\right]
\end{aligned}
$$

for any sufficiently small $\delta>0$ and $c=c(\delta)>0$. Since $\phi$ vanishes at infinity, the dominated convergence theorem shows that after reducing $\delta$ (if necessary) each term in the numerator of (4.47) involving $\alpha \in\{2, \ldots, L\}$ tends to zero as $n \rightarrow \infty$. Handling the other terms in (4.47) as in the proof of Theorem 2.6, we obtain (4.42).

V. Proofs of Theorems 2.3, 2.7 and extensions (simply degenerate case). The plan of this section is to prove Theorems 2.3 and 2.7 together with extensions of Theorems 2.5 and 2.7. The extensions cover the case where $G$ has nonunique isolated minimum points of which some are simply degenerate and the rest nondegenerate. These extensions are stated and proved as Theorem 5.5 at the end of this section.

The proof of Theorem 2.3 depends on Lemmas 4.1, 4.3, and on three additional lemmas, Lemmas 5.1-5.3, which are stated just below. Lemma 5.1 states facts about the function $\Phi(Y)$ defined implicitly by (2.11). Lemma 5.2 concerns the operator $\tau \rho A \rho \tau$ which appears in the definition of the determinant $\tilde{\Delta}$ in Theorem 2.3. Lemma 5.3 is the analogue, in the simply degenerate case, of Lemma 4.2. After stating the lemmas, we prove Theorem 2.3 , then prove the lemmas.

Given $Y^{*}$ a simply degenerate minimum point of $G$, we recall that $\tau$ is the orthogonal projection onto $\mathscr{K}:=\operatorname{ker}\left(G^{\prime \prime}\left(Y^{*}\right)\right), U$ is a unit vector in $\mathscr{K}$, and $\rho$ is the bounded operator on $\mathcal{H}$ defined by

$$
\rho Y:=Y-[\langle A U, Y\rangle /\langle A U, U\rangle] U .
$$


As explained in §II (see (2.9)-(2.10)), equation (5.4) in the next lemma implies the vanishing of the cross terms in $G\left(Y^{*}+Y\right)$ when $Y$ is written in the form (2.8).

LemMa 5.1. Suppose that $Y^{*}$ is a simply degenerate minimum point of $G$. Define $F_{3}=F_{3}\left(Y^{*} ; \cdot\right)$ by (2.3). Then there exists $\bar{\delta}>0$ such that the equation

$$
\Phi(V)=-B_{\perp} \tau F_{3}^{\prime}(V+\Phi(V))
$$

has a unique solution $\Phi(V)=\Phi\left(Y^{*} ; V\right)$ for all $V \in S(0, \bar{\delta})$. For all such $V, \Phi(V)$ is $C^{\infty}, \Phi(V) \in K^{\perp} \cap \mathscr{D}\left(A^{-1}\right)$, and

$$
\|\Phi(V)\|=O\left(\|V\|^{2}\right) \text { as }\|V\| \rightarrow 0 .
$$

For $z$ real and $|z|<\bar{\delta}$, we also have for all $X \in \mathcal{K}^{\perp}$

$$
\begin{aligned}
\left\langle G^{\prime}\left(Y^{*}+z U+\Phi(z U)\right), X\right\rangle:= & \left\langle A^{-1}\left(Y^{*}+z U+\Phi(z U)\right), X\right\rangle \\
& +\left\langle F^{\prime}\left(Y^{*}+z U+\Phi(z U)\right), X\right\rangle=0 .
\end{aligned}
$$

LĖMMA 5.2. The operator $\tau A^{-1} \tau$, with domain $\mathcal{K}^{\perp} \cap \mathscr{D}\left(A^{-1}\right)$, is invertible on $\mathcal{K}^{\perp}$ with inverse given by $A_{\perp}:=\tau \rho A \rho \tau . A_{\perp}$ is the covariance operator of a mean zero Gaussian measure $P_{A_{\perp}}$ on $\mathcal{K}^{\perp}$.

Let $\bar{\delta}$ be the number in Lemma 5.1. For the next lemma, we define

$$
W(z)=W\left(Y^{*} ; z\right):=z U+\Phi\left(Y^{*} ; z U\right)
$$

for $z$ real and $|z|<\bar{\delta}$. Since by Lemma 5.1 the function $z \rightarrow \Phi(z U)$ is well defined and smooth for such $z, W(z)$ shares these properties. For $\delta \in(0, \bar{\delta}), n \in\{1,2, \ldots\}$, and $|z|<\delta n^{1 / k}$, we define the set $S(\delta, n, z)=S\left(Y^{*} ; \delta, n, z\right)$ by

$$
S(\delta, n, z):=\left\{X: X \in \mathcal{K}^{\perp},\left\|W\left(z / n^{1 / k}\right)+X / \sqrt{n}\right\|<\delta\right\} .
$$

This set is well defined and nonempty. Finally, for the same $\delta, n, z$ and for $X \in \mathfrak{K}^{\perp}$, we define $\Omega_{n}(z, X)=\Omega_{n}\left(Y^{*} ; z, X\right)$ by (2.19).

LEMMA 5.3. Suppose that $Y^{*}$ is an isolated, simply degenerate minimum point of $G$. Then

$$
\tilde{\Delta}=\tilde{\Delta}\left(Y^{*}\right):=\operatorname{det}\left(I+\tau \rho A \rho \tau F^{\prime \prime}\left(Y^{*}\right) \tau\right)=\operatorname{det}\left(I+A_{\perp} \tau F^{\prime \prime}\left(Y^{*}\right) \tau\right)
$$

is well defined and $\tilde{\Delta}>0$. Also $\tau G^{\prime \prime}\left(Y^{*}\right) \tau$ is invertible on $\mathcal{K}^{\perp}$ with inverse operator denoted by $B_{\perp}=B_{\perp}\left(Y^{*}\right) . B_{\perp}$ is the covariance operator of a mean zero Gaussian measure $P_{B_{\perp}}$ on $\mathcal{K}^{\perp}$. Consider the type $k=k\left(Y^{*}\right)$ and the strength $\lambda=\lambda\left(Y^{*}\right)$ defined by (2.13)-(2.14). If $k<\infty$, then $k$ is an even integer, $k \geqslant 4$, and $\lambda>0$. Let $\bar{\delta}$ be the number in Lemma 5.1. We have for all $\delta \in(0, \bar{\delta}]$

$$
\begin{gathered}
e^{n G^{*}} \int_{S\left(\sqrt{ } n Y^{*}, n n \delta\right)} \psi(Y / \sqrt{n}) e^{-n F(Y / \sqrt{ } n)} d P_{A}(Y)=[2 \pi\langle A U, U\rangle \tilde{\Delta}]^{-1 / 2} n^{1 / 2-1 / k} \\
\cdot \int_{|z|<\delta n^{1 / k}} \int_{S(\delta, n, z)} \Omega_{n}\left(z / n^{1 / k}, X / \sqrt{n}\right) d P_{B_{\perp}}(X) e^{-\lambda z^{k}} d z .
\end{gathered}
$$

REMARK 5.3(a). The only smoothness required of $\psi$ in (5.8) is that it be continuous. This form of Lemma 5.3 is used below in the proofs of Theorems 2.7 and 5.5. 
Proof of Theorem 2.3. The facts about $\tilde{\Delta}, B_{\perp}, P_{B_{\perp}}$ are proved in Lemma 5.3. We prove (2.20) by showing that there exist functionals $\left\{\Gamma_{j, k} ; j=0,1, \ldots\right\}$ such that for any integer $M \geqslant 0$

$$
e^{n G^{*}} J_{n}=n^{1 / 2-1 / k}\left\{\sum_{j=0}^{M} \Gamma_{j, k} n^{-2 j / k}+O\left(n^{-2(M+1) / k}\right)\right\} \quad \text { as } n \rightarrow \infty .
$$

The $\left\{\Gamma_{j, k}\right\}$ are found by expanding the integrand $\Omega_{n}\left(z / n^{1 / k}, X / \sqrt{n}\right)$ in (2.20) in powers of $z / n^{1 / k}$ and $X / \sqrt{n}=X / n^{(k / 2) / k}$ and then by integrating term-by-term with respect to $e^{-\lambda z^{k}} d z d P_{B_{\perp}}$ over $\mathbf{R} \times \mathcal{K}^{\perp}$. Since $e^{-\lambda z^{k}}$ is an even density and $P_{B_{\perp}}$ is mean zero, only integral powers of $n^{-2 / k}$ survive.

We first prove facts about $S(\delta, n, z)$ which are needed below. Let $\bar{\delta}$ be the number in Lemma 5.1. Pick $\delta \in(0, \bar{\delta})$ and $|z|<\delta n^{1 / k}$. Since $U \in \mathcal{K},\|U\|=1, \Phi\left(z U / n^{1 / k}\right)$ $\in \mathcal{K}^{\perp}, X \in \mathcal{K}^{\perp}$, we see that

$$
S(\delta, n, z)=\left\{X: X \in \mathcal{K}^{\perp},\left\|\Phi\left(z U / n^{1 / k}\right)+X / \sqrt{n}\right\|^{2}<\delta^{2}-\left(z / n^{1 / k}\right)^{2}\right\} .
$$

By (5.3) $\left\|\Phi\left(z U / n^{1 / k}\right)\right\|=O\left(\left|z / n^{1 / k}\right|^{2}\right)=O\left(\delta^{2}\right)$ as $\delta \rightarrow 0$. Hence there exists $\delta_{1} \in$ $(0, \bar{\delta}]$ such that if $\delta \in\left(0, \delta_{1}\right]$, then

$$
\begin{gathered}
\left\|W\left(z / n^{1 / k}\right)\right\| \leqslant 2 \delta, \\
S(\delta, n, z) \subset\left\{X: X \in \mathcal{K}^{\perp},\|X / \sqrt{n}\|<2 \delta\right\},
\end{gathered}
$$

and if in addition $\left|z / n^{1 / k}\right|<\delta / 2$, then

$$
S(\delta, n, z) \supset\left\{X: X \in \mathscr{K}^{\perp},\|X / \sqrt{n}\|<\delta / 2\right\} .
$$

We start the proof of (2.21). By Lemmas 4.1, 5.3, there exists $\bar{\delta}>0$ such that for any $\delta \in(0, \bar{\delta}]$

$$
\begin{aligned}
& e^{n G^{*}} J_{n}=\gamma n^{1 / 2-1 / k} \int_{|z|<\delta n^{1 / k}} \int_{S(\delta, n, z)} \psi_{n} \\
& \cdot \exp \left[-n\left\{G\left(Y^{*}+W\left(z / n^{1 / k}\right)\right)-G\left(Y^{*}\right)-\lambda\left(z / n^{1 / k}\right)^{k}\right\}\right] \\
& \cdot e^{-n F_{3, n}} d P_{B_{\perp}}(X) e^{-\lambda z^{k}} d z+O\left(e^{-n c}\right) .
\end{aligned}
$$

Here $c=c(\delta)>0, \gamma:=[2 \pi\langle A U, U\rangle \tilde{\Delta}]^{-1 / 2}, \psi_{n}:=\psi\left(Y^{*}+W\left(z / n^{1 / k}\right)+X / \sqrt{n}\right)$, and $\tilde{F}_{3, n}:=\tilde{F}_{3}\left(W\left(z / n^{1 / k}\right), X / \sqrt{n}\right)$. We show how to go from (5.14) to (2.20). Let $\delta_{1}$ be the number defined before (5.11). Since $\psi$ and $F$ are $C^{\infty}$, we see that $W\left(z / n^{1 / k}\right)$, $\psi\left(Y^{*}+W\left(z / n^{1 / k}\right)+X / \sqrt{n}\right), G\left(Y^{*}+W\left(z / n^{1 / k}\right)\right)$, and $\tilde{F}_{3}\left(W\left(z / n^{1 / k}\right), X / \sqrt{n}\right)$ are $C^{\infty}$ for $\delta \in\left(0, \delta_{1}\right],|z|<\delta n^{1 / k}$, and $X \in S(\delta, n, z)$. Let an integer $M \geqslant 0$ be given. (If $M=0$, then the sums in (5.19), (5.20), (5.21) below are absent.) Since $W(0)=0$, there exist elements $\left\{V_{i} ; i=1,2, \ldots\right\}$ in $\mathcal{H}$ such that

$$
W\left(z / n^{1 / k}\right)=\sum_{i=1}^{2 M+1} V_{i}\left(z / n^{1 / k}\right)^{i}+O\left(z^{2 M+2} n^{-2(M+1) / k}\right) \quad \text { as }\left|z / n^{1 / k}\right| \rightarrow 0
$$


For sufficiently small $\delta_{2} \in\left(0, \delta_{1}\right]$ and for any $\delta \in\left(0, \delta_{2}\right],|z|<\delta n^{1 / k}, X \in$ $S(\delta, n, z)$, we claim that there exist multilinear functionals $\left\{a_{i j} ; i, j=0,1, \ldots\right\}$ such that

$$
\begin{aligned}
\psi\left(Y^{*}+W\left(z / n^{1 / k}\right)+X / \sqrt{n}\right)= & \sum_{0 \leqslant i+j \leqslant 2 M+1}\left(z / n^{1 / k}\right)^{i} a_{i j}\left((X / \sqrt{n})^{j}\right) \\
& +O\left(z^{2 m+2} n^{-2(M+1) / k}+\|X\|^{2 M+2} n^{-(M+1)}\right) \\
& \text { as }\left|z / n^{1 / k}\right|+\|X / \sqrt{n}\| \rightarrow 0 .
\end{aligned}
$$

We explain how to derive (5.16). We may pick $\delta_{2} \in\left(0, \delta_{1}\right]$ so small that $\left\|D^{2 M+2} \psi\left(Y^{*}+Y\right)\right\|$ is uniformly bounded on the set $S\left(0,4 \delta_{2}\right)$. By (5.11)-(5.12), for any $\delta \in\left(0, \delta_{2}\right], S\left(0,4 \delta_{2}\right)$ contains the set

$$
\left\{Y: Y=s\left(W\left(z / n^{1 / k}\right)+X / \sqrt{n}\right),|z|<\delta n^{1 / k}, X \in S(\delta, n, z), 0 \leqslant s \leqslant 1\right\} .
$$

Hence by Taylor's theorem and the bound $\left\|W\left(z / n^{1 / k}\right)\right\|=O\left(z / n^{1 / k}\right)$, the left-hand side of (5.16) equals

$$
\sum_{j=0}^{2 M+1} D^{j} \psi\left(Y^{*}\right)\left(W\left(z / n^{1 / k}\right)+X / \sqrt{n}\right)^{j}+O\left(\left\{\left|z / n^{1 / k}\right|+\|X / \sqrt{n}\|\right\}^{2 M+2}\right) .
$$

Because of (5.15), (5.18) can be put in the form of the right-hand side of (5.16). We next expand the other terms in (5.14). By the definition (2.14)(a) of $k$ and by (5.15), there exist functionals $\left\{g_{i} ; i=1,2, \ldots\right\}$ such that

$$
\begin{aligned}
& n\left[G\left(Y^{*}+W\left(z / n^{1 / k}\right)\right)-G\left(Y^{*}\right)-\lambda\left(z / n^{1 / k}\right)^{n}\right] \\
& \quad=\sum_{i=1}^{2 M+1} g_{i} z^{k+i} / n^{i / k}+O\left(|z|^{k+2 M+2} n^{-2(M+1) / k}\right) \quad \text { as }\left|z / n^{1 / k}\right| \rightarrow 0 .
\end{aligned}
$$

To handle the $n \tilde{F}_{3, n}$ term in (5.14), we pick $\delta_{3} \in\left(0, \delta_{2}\right]$ so small that $\left\|D^{2 M+4} F\left(Y^{*}+Y\right)\right\|$ is uniformly bounded on $S(0,4 \delta)$. Then for $\delta \in\left(0, \delta_{3}\right],|z|<$ $\delta n^{1 / k}, X \in S(\delta, n, z)$, we have

$$
\begin{aligned}
& n \tilde{F}_{3}\left(W\left(z / n^{1 / k}\right), X / \sqrt{n}\right) \\
& =\int_{0}^{1}\left\langle\left\{F^{\prime \prime}\left(Y^{*}+W\left(z / n^{1 / k}\right)+s X / \sqrt{n}\right)-F^{\prime \prime}\left(Y^{*}\right)\right\} X, X\right\rangle(1-s) d s \\
& =\sum_{i=1}^{2 M+1}(1 / i !) \int_{0}^{1}\left\{D^{2+i} F\left(Y^{*}\right)\left(\left[W\left(z / n^{1 / k}\right)+s X / \sqrt{n}\right]^{i}, X^{2}\right)\right\}(1-s) d s \\
& \quad+O\left(\left[\left\|W\left(z / n^{1 / k}\right)\right\|^{2 M+2}+\|X / \sqrt{n}\|^{2 M+2}\right]\|X\|^{2}\right) \\
& \text { as }\left|z / n^{1 / k}\right|+\|X / \sqrt{n}\| \rightarrow 0 .
\end{aligned}
$$

Hence there exist multilinear functionals $\left\{f_{i j} ; i=1,2, \ldots, j=2,3, \ldots\right\}$ such that

$$
\begin{aligned}
& n \tilde{F}_{3}\left(W\left(z / n^{1 / k}\right), X / \sqrt{n}\right)=n \sum_{\substack{i \geqslant 1, j \geqslant 2 \\
i+j \leqslant 2 M+3}}\left(z / n^{1 / k}\right)^{i} f_{i j}\left((X / \sqrt{n})^{j}\right) \\
& +O\left(\left[|z|^{2 M+2} n^{-2(M+1) / k}+\|X\|^{2 M+2} n^{-(M+1)}\right]\|X\|^{2}\right) \\
& \text { as }\left|z / n^{1 / k}\right|+\|X / \sqrt{n}\| \rightarrow 0 \text {. }
\end{aligned}
$$


We derive a bound that will be used below. By (5.21) with $M=0$, we have for $|z|<\delta n^{1 / k}, X \in S(\delta, n, z)$,

$$
\begin{aligned}
n\left|\tilde{F}_{3}\left(W\left(z / n^{1 / k}\right), X / \sqrt{n}\right)\right|=O\left(\left[\left|z / n^{1 / k}\right|\right.\right. & \left.+\|X / \sqrt{n}\|]\|X\|^{2}\right) \\
& \text { as }\left|z / n^{1 / k}\right|+\|X / \sqrt{n}\| \rightarrow 0 .
\end{aligned}
$$

By (5.18), we conclude that for such $z, X$

$$
n\left|\tilde{F}_{3}\left(W\left(z / n^{1 / k}\right), X / \sqrt{n}\right)\right|=O\left(\delta\|X\|^{2}\right) \quad \text { as }\left|z / n^{1 / k}\right|+\|X / \sqrt{n}\| \rightarrow 0 .
$$

This shows that the term $n \tilde{F}_{3}\left(W\left(z / n^{1 / k}\right), X \sqrt{n}\right)$ in $(2.15)-(2.16)$ is an error term.

We now return to the proof of (2.20). The next step is to substitute (5.16), (5.19), (5.21) into (5.14), where $\delta \in\left(0, \delta_{3}\right]$. As in the nondegenerate case, there exists an integer $N=N(M)$ and for each $m \in\{0, \ldots, 2 M+1\}$ there exists a functional $\Xi_{m, k}(z, X)$ such that

$$
\begin{aligned}
e^{n G^{*}} J_{n}= & \gamma n^{1 / 2-1 / k} \sum_{m=0}^{2 M+1} n^{-m / k} \\
& \cdot \int_{|z|<\delta n^{1 / k}} \int_{S(\delta, n, z)} \Xi_{m, k}(z, X) d P_{B_{\perp}}(X) e^{-\lambda z^{k}} d z+T_{M, k}(n),
\end{aligned}
$$

where

$$
\begin{aligned}
\left|T_{M, k}(n)\right| \leqslant \text { const }\left\{e^{-n c}+n^{-2(M+1) / k}\right. & \int_{|z|<\delta n^{1 / k}} \int_{S(\delta, n, z)}\left[1+|z|^{N(M)}+\|X\|^{N(M)}\right] \\
\cdot \exp \left[n \mid G\left(Y^{*}+\right.\right. & \left.W\left(z / n^{1 / k}\right)\right)-G\left(Y^{*}\right) \\
& \left.\left.-\lambda\left(z / n^{1 / k}\right)^{k}|+n| \tilde{F}_{3, n} \mid\right] d P_{B_{\perp}}(X) e^{-\lambda z^{k}} d z\right\} .
\end{aligned}
$$

We have $\Xi_{0, k}=\psi\left(Y^{*}\right)$. For each $m \in\{1, \ldots, 2 M+1\}, \Xi_{m, k}$ is a sum of products of terms involving $z$ and $X$. As an aid to understanding, we point out one contribution to $\Xi_{m, k}$. Taking the zeroth order contributions from the two exponentials in (5.14), we see that the term $\left(z / n^{1 / k}\right)^{i} a_{i j}\left((X / \sqrt{n})^{j}\right)$ from (5.16) is a summand in $\Xi_{m, k}$ if $i+j k / 2=m$. In general, each summand in $\Xi_{m, k}$ contains $i z$ 's and $j X$ 's with $i$ and $j$ related to $m$ in this same way. We see that if $m$ is odd, then either $i$ or $j$ must be odd and $\Xi_{m, k}$ must be an odd function of $z$ or $X$.

We now show how to go from (5.24) to (5.9). We claim that for each $m \in$ $\{0, \ldots, 2 M+1\}$

$$
\int_{|z|<\delta n^{1 / k}} \int_{S(\delta, n, z)} \Xi_{m, k} d P_{B_{\perp}} e^{-\lambda z^{k}} d z=\int_{\mathbf{R}} \int_{\mathscr{K} \mathcal{K}^{\perp}} \Xi_{m, k} d P_{B_{\perp}} e^{-\lambda z^{k}} d z+O\left(e^{-n c_{1}}\right)
$$

for some constant $c_{1}>0$ and that for sufficiently small $\delta \in\left(0, \delta_{3}\right]$

$$
\left|T_{M, k}(n)\right|=O\left(n^{-2(M+1) / k}\right) \text {. }
$$


If $m$ is odd, then $\int_{\mathbf{R}} \int_{\mathcal{K}^{\perp}} \Xi_{m, k} d P_{B_{\perp}} e^{-\lambda z^{k}} d z=0$ since $\Xi_{m, k}$ is an odd function of either $z$ or $X$. Substituting (5.26)-(5.27) into (5.24), we obtain the expansion (5.9) with

$$
\Gamma_{j, k}=\gamma \int_{\mathbf{R}} \int_{\mathcal{K K}^{\perp}} \Xi_{2 j, k}(z, X) d P_{B_{\perp}}(X) e^{-\lambda z^{k}} d z .
$$

We prove (5.26). For each $m \in\{0, \ldots, 2 M+1\}$ there exists an integer $N_{m} \geqslant 0$ such that

$$
\left|\Xi_{m, k}(z, X)\right| \leqslant \operatorname{const}\left(1+|z|^{N_{m}}\right)\left(1+\|X\|^{N_{m}}\right)
$$

By (5.13),

$$
\begin{aligned}
\{(z, X):|z|<\delta & \left.n^{1 / k}, X \in S(\delta, n, z)\right\}^{c} \\
& \subset\left\{(z, X):|z| \geqslant \delta n^{1 / k} / 2, X \in \mathscr{K} \mathcal{K}^{\perp}\right\} . \\
& \cup\left\{(z, X):|z|<\delta n^{1 / k} / 2, X \in \mathcal{K}^{\perp},\|X\| \geqslant \delta \sqrt{n} / 2\right\},
\end{aligned}
$$

where the superscript $c$ denotes complement in $\mathbf{R} \times \mathcal{K}^{\perp}$. By (5.29)-(5.30)

$$
\begin{aligned}
& \left|\int_{|z|<\delta n^{1 / k}} \int_{S(\delta, n, z)} \Xi_{m, k} d P_{B_{\perp}} e^{-\lambda z^{k}} d z-\int_{\mathbf{R}} \int_{\mathscr{K} \mathcal{K}^{\perp}} \Xi_{m, k} d P_{B_{\perp}} e^{-\lambda z^{k}} d z\right| \\
& \leqslant \text { const }\left[\int_{|z|>\delta n^{1 / k} / 2}\left(1+|z|^{N_{m}}\right) e^{-\lambda z^{k}} d z \int_{\mathscr{K}^{\perp}}\left(1+\|X\|^{N_{m}}\right) d P_{B_{\perp}}(X)\right. \\
& \left.\quad+\int_{\mathbf{R}}\left(1+|z|^{N_{m}}\right) e^{-\lambda z^{k}} d z \int_{\left\{X: X \in \mathcal{K}^{\perp},\|X\| \geqslant \delta, n / 2\right\}}\left(1+\|X\|^{N_{m}}\right) d P_{B_{\perp}}(X)\right] .
\end{aligned}
$$

By the strict positivity of $\lambda$, the evenness of $k$, and Lemma 4.3 applied to $P:=P_{B_{\perp}}$, the right-hand side of (5.31) is bounded by const $\exp \left(-n c_{1}\right)$ for some $c_{1}>0$. This yields (5.26).

We prove (5.27). By (5.19) with $M=0$, we have for $|z|<\delta n^{1 / k}$

$$
\begin{gathered}
n\left|G\left(Y^{*}+W\left(z / n^{1 / k}\right)\right)-G\left(Y^{*}\right)-\lambda\left(z / n^{1 / k}\right)^{k}\right|=O\left(|z|^{k+1} / n^{1 / k}\right) \\
=O\left(\delta|z|^{k}\right) \text { as }\left|z / n^{1 / k}\right| \rightarrow 0 .
\end{gathered}
$$

By (5.23), (5.32) there exists a constant $c_{2}>0$ such that

$$
\begin{aligned}
&\left|T_{M, k}(n)\right| \leqslant \operatorname{const}\left[e^{-n c}+n^{-2(M+1) / k}\right. \\
& \cdot \int_{\mathbf{R}} \int_{\mathscr{K}^{\perp}}\left[1+|z|^{N(M)}+\|X\|^{N(M)}\right] \\
&\left.\cdot e^{c_{2} \delta\|X\|^{2}} d P_{B_{\perp}}(X) e^{c_{2} \delta|z|^{k}} e^{-\lambda z^{k}} d z\right] .
\end{aligned}
$$

By the strict positivity of $\lambda$, the evenness of $k$, and Lemma 4.3 applied to $P:=P_{B_{\perp}}$, the integrals in (5.33) are finite for all sufficiently small $\delta \in\left(0, \delta_{3}\right]$. This yields (5.27). 
We next prove Lemmas 5.1-5.3.

Proof of Lemma 5.1. We first prove existence and properties of $\Phi(V)$. The formula

$$
\begin{aligned}
R(V, \Phi): & =\Phi+B_{\perp} \tau F_{3}^{\prime}(V+\Phi) \\
& =\Phi+B_{\perp} \tau\left[F^{\prime}\left(Y^{*}+V+\Phi\right)-F^{\prime}\left(Y^{*}\right)-F^{\prime \prime}\left(Y^{*}\right)(V+\Phi)\right]
\end{aligned}
$$

defines a $C^{\infty}$ mapping $R$ of $\mathscr{H} \times \mathcal{H}$ into $\mathcal{H}$. We have $R(0,0)=0$ and $D_{\Phi} R(0,0)=I$, where $D_{\Phi}$ denotes partial differentiation with respect to $\Phi$. By the implicit function theorem [Berger, Theorem 3.1.10 and Remark], there exists a unique $C^{\infty}$ mapping $\Phi(V)$ defined in a neighborhood $\mathcal{V}$ of 0 such that $\Phi(0)=0$ and $R(V, \Phi(V))=0$ for $V \in \mathfrak{V} . \Phi$ maps $\mathscr{V}$ into $\mathcal{K}^{\perp} \cap \mathscr{D}\left(A^{-1}\right)$ since $\Phi(V)=-B_{\perp} \tau F_{3}^{\prime}(V+\Phi(V))$ and $\mathscr{R}\left(\mathscr{B}_{\perp}\right)$ $\subset \mathscr{D}\left(\tau G^{\prime \prime}\left(Y^{*}\right) \tau\right) \subset \mathcal{K}^{\perp} \cap \mathscr{D}\left(A^{-1}\right)$. The bound (5.3) follows since $D \Phi(0)=0$.

We now prove (5.4). Substituting $Y:=z U$ in (5.2) and writing $\Phi$ for $\Phi(z U), W$ for $(z U+\Phi(z U))$, we find for any $X \in \mathscr{K}^{\perp}$ (so that $\tau X=X$ )

$$
\begin{aligned}
& -\left\langle A^{-1} \Phi, X\right\rangle-\left\langle F^{\prime \prime}\left(Y^{*}\right) \Phi, X\right\rangle=-\left\langle B_{\perp}^{-1} \Phi, X\right\rangle \\
& =\left\langle F_{3}^{\prime}(W), X\right\rangle=\left\langle F^{\prime}\left(Y^{*}+W\right)-F^{\prime}\left(Y^{*}\right)-F^{\prime \prime}\left(Y^{*}\right) W, X\right\rangle .
\end{aligned}
$$

From (5.35), we conclude that

$$
\left\langle F^{\prime}\left(Y^{*}+W\right), X\right\rangle=\left\langle F^{\prime}\left(Y^{*}\right), X\right\rangle+\left\langle F^{\prime \prime}\left(Y^{*}\right)(W-\Phi) X\right\rangle-\left\langle A^{-1} \Phi, X\right\rangle .
$$

Since $G^{\prime}\left(Y^{*}\right)=0, G^{\prime \prime}\left(Y^{*}\right) U=0$, we see that

$$
F^{\prime}\left(Y^{*}\right)=-A^{-1} Y^{*}, \quad F^{\prime \prime}\left(Y^{*}\right)(W-\Phi)=F^{\prime \prime}\left(Y^{*}\right)(z U)=-A^{-1}(z U) .
$$

Thus the right-hand side of (5.36) equals $-\left\langle A^{-1}\left(Y^{*}+W\right), X\right\rangle$. This proves (5.4).

Proof of Lemma 5.2. To prove the first assertion, it suffices to prove that for any $X \in \mathcal{K}^{\perp}$

$$
\tau A^{-1} \tau \rho A \rho \tau X=X .
$$

For $X \in \mathcal{K}^{\perp}, \tau X=X$, and since $\tau U=0$, we have $\tau \rho V=\tau V=V-\langle V, U\rangle U$ for $V \in \mathscr{H}$. Thus,

$$
\begin{aligned}
\tau A^{-1} \tau \rho A \rho \tau X & =\tau A^{-1} \tau A \rho X=\tau A^{-1}(A \rho X-\langle A \rho X, U\rangle U) \\
& =\tau \rho X-\langle A \rho X, U\rangle \tau A^{-1} U=X-\langle\rho X, A U\rangle \tau A^{-1} U .
\end{aligned}
$$

Thus, (5.38) is proved once we show $\langle\rho X, A U\rangle=0$. The latter is easily checked from (5.1). $A_{\perp}$ is the covariance operator of a mean zero Gaussian measure $P_{A_{\perp}}$ since $A_{\perp}$ is positive, symmetric, and trace class [Gihman-Skorohod, Theorem V.6.1]. By [Rajput] the support of $P_{A_{\perp}}$ equals the closure of the range of $A_{\perp}$, which is $\mathcal{K}^{\perp}$.

Proof of Lemma 5.3. The assertions about $\tilde{\Delta}$ and $B_{\perp}$ follow from Lemma 4.4. Indeed, by definition of simple degeneracy and Lemma 5.2, the hypotheses of this lemma are satisfied with $\mathcal{K}_{1}:=\mathscr{K}^{\perp}, A_{1}:=\tau \rho A \rho \tau$, and $\Lambda:=\tau F^{\prime \prime}\left(Y^{*}\right) \tau$. In addition, (4.28) implies that for $X \in \mathcal{K}^{\perp}$ (so that $\tau X=X$ )

$$
\begin{aligned}
d P_{B_{\perp}}(X) & =\sqrt{\tilde{\Delta}} \exp \left(-(1 / 2)\left\langle\tau F^{\prime \prime}\left(Y^{*}\right) X, X\right\rangle\right) d P_{A_{\perp}}(X) \\
& =\sqrt{\tilde{\Delta}} \exp \left(-(1 / 2)\left\langle F^{\prime \prime}(Y)^{*} X, X\right\rangle\right) d P_{A_{\perp}}(X) .
\end{aligned}
$$


We next prove the assertions about $k$ and $\lambda$. Consider the number $\bar{\delta}$ in Lemma 5.1. For $|z|<\bar{\delta}, \Phi(z U)$ is well defined and smooth and

$$
\|\Phi(z U)\|=O\left(z^{2}\right) \quad \text { as }|z| \rightarrow 0 .
$$

Since $Y^{*}$ is an isolated minimum point of $G$ and $\Phi(z U) \neq-z U$ for $z$ sufficiently small (by (5.40)), we have for all such $z$

$$
0<G\left(Y^{*}+z U+\Phi(z U)\right)-G\left(Y^{*}\right)=\lambda z^{k}+O\left(z^{k+1}\right) .
$$

We conclude that $k$ is even and $\lambda>0$. We next prove $k>2$. Since $U, \Phi(z U) \in$ $\mathscr{D}\left(A^{-1}\right)$, we have by (3.6) and (5.40)

$$
\begin{aligned}
G\left(Y^{*}+z U\right. & +\Phi(z U))=G\left(Y^{*}\right)+\left\langle G^{\prime}\left(Y^{*}\right), z U+\Phi(z U)\right\rangle \\
& +(1 / 2)\left\langle G^{\prime \prime}\left(Y^{*}\right)(z U+\Phi(z U)),(z U+\Phi(z U))\right\rangle+O\left(z^{3}\right) \\
= & G\left(Y^{*}\right)+(1 / 2) z^{2}\left\langle G^{\prime \prime}\left(Y^{*}\right) U, U\right\rangle+O\left(z^{3}\right) .
\end{aligned}
$$

But $G^{\prime \prime}\left(Y^{*}\right) U=0$ and comparison of (5.42) with (2.13) shows that $k>2$.

In order to prove (5.8), we need to know the joint distribution of $\langle Y, U\rangle$ and $\tau Y$, where $Y$ is an $\mathcal{G}$-valued random variable with distribution $P_{A}$. This joint distribution is given in the next lemma, which will be proved after the completion of the proof of Lemma 5.3.

Lemma 5.4. Given $Y \in \mathcal{H}$, define $z(Y):=\langle Y, U\rangle$. For any bounded integrable function $f: \mathcal{K} \times \mathcal{K}^{\perp} \rightarrow \mathbf{R}$, we have

$$
\begin{aligned}
& \int f(z(Y) U, \tau Y) d P_{A}(Y) \\
& \quad=\frac{1}{(2 \pi\langle A U, U\rangle)^{1 / 2}} \int_{\mathbf{R} \times \mathcal{K}^{\perp}} f(z U, X) \exp \left[-I(z U)-z\left\langle A^{-1} U, X\right\rangle\right] d z d P_{A_{\perp}}(x) .
\end{aligned}
$$

The measure $P_{A_{\perp}}$ is defined in Lemma 5.2.

We now prove (5.8) by showing that for all $\delta \in(0, \bar{\delta})$

$$
\begin{aligned}
& e^{n G^{*}} \int_{\left\{Y:\left\|Y / \sqrt{ }-Y^{*}\right\|<\delta\right\}} \psi(Y / \sqrt{n}) e^{-n F(Y / \sqrt{ } n)} d P_{A}(Y) \\
& \quad=[2 \pi\langle A U, U\rangle \tilde{\Delta}]^{-1 / 2} \int_{|z|<\delta \sqrt{ } n} \int_{S_{n}(\delta, z)} \Omega_{n}(z / \sqrt{n}, X / \sqrt{n}) d P_{B_{\perp}}(X) e^{-n \lambda(z / \sqrt{ } n)^{k}} d z,
\end{aligned}
$$

where $S_{n}(\delta, z):=\left\{X: X \in \mathscr{K}^{\perp},\|W(z / \sqrt{n})+X / \sqrt{n}\|<\delta\right\}$. Scaling $z \rightarrow z n^{1 / 2-1 / k}$ completes the proof. By Lemma 4.5, the left-hand side of (5.44) equals

$$
e^{n G^{*}} \int_{S(0, n n \delta)} \psi\left(Y^{*}+Y / \sqrt{n}\right) e^{-n F\left(Y^{*}+Y / \sqrt{ } n\right)} e^{-n I\left(Y^{*}\right)-\sqrt{ }\left\langle A^{-1} Y^{*}, Y\right\rangle} d P_{A}(Y)
$$


We write (5.45) as an iterated integral using Lemma 5.4 and obtain

$$
\begin{aligned}
& \frac{e^{n G^{*}}}{[2 \pi\langle A U, U\rangle]^{1 / 2}} \int_{|z|<\delta, n} \int_{\|(z U+X) / \sqrt{ } n\|<\delta} \psi\left(Y^{*}+\frac{z U+X}{\sqrt{n}}\right) \\
& \quad \cdot \exp \left[-n F\left(Y^{*}+\frac{z U+X}{\sqrt{n}}\right)\right] \\
& \quad \cdot \exp \left[-n I\left(Y^{*}\right)-\sqrt{n}\left\langle A^{-1} Y^{*}, z U+X\right\rangle-I(z U)-\left\langle A^{-1}(z U), X\right\rangle\right] d P_{A_{\perp}}(X) d z .
\end{aligned}
$$

For fixed $z$ satisfying $|z|<\delta \sqrt{n}$, we have $\Phi(z U / \sqrt{n}) \in \mathscr{K}^{\perp}$ and so we may change variables $X \rightarrow X+\sqrt{n} \Phi(z U / \sqrt{n})$ in the inner integral in (5.46). For the rest of this proof we write $\Phi$ and $W$ instead of $\Phi(z U / \sqrt{n})$ and $W(z / \sqrt{n}):=z U / \sqrt{n}+$ $\Phi\left(z U / \sqrt{n}\right.$ in order to save space. By Lemma 5.2, $\Phi \in \mathscr{D}\left(A_{\perp}^{-1}\right)$ and so by Lemma 4.5, (5.46) equals

$$
\begin{aligned}
& \frac{e^{n G^{*}}}{[2 \pi\langle A U, U\rangle]^{1 / 2}} \int_{|z|<\delta, n} \int_{S_{n}(\delta, z)} \psi\left(Y^{*}+W+X / \sqrt{n}\right) \exp \left[-n F\left(Y^{*}+W+X / \sqrt{n}\right)\right] \\
& \cdot \exp \left[-n I\left(Y^{*}\right)-\sqrt{n}\left\langle A^{-1} Y^{*}, \sqrt{n} W+X\right\rangle-I(z U)\right. \\
& \left.\quad-\left\langle A^{-1}(z U), X+\sqrt{n} \Phi\right\rangle-(1 / 2) n\left\langle A_{\perp}^{-1} \Phi, \Phi\right\rangle-\sqrt{n}\left\langle A^{-1} \Phi, X\right\rangle\right] d P_{A_{\perp}}(X) d z .
\end{aligned}
$$

Since $\Phi$ and $X$ are in $\mathcal{K}^{\perp}$, we can replace $A_{\perp}^{-1}$ in (5.47) by $A^{-1}$, and after collecting terms, (5.47) becomes

$$
\begin{aligned}
\frac{e^{n G^{*}}}{[2 \pi\langle A U, U\rangle]^{1 / 2}} & \int_{|z|<\delta, n} \int_{S_{n}(\delta, z)} \psi\left(Y^{*}+W+X / \sqrt{n}\right) \exp \left[-n F\left(Y^{*}+W+X / \sqrt{n}\right)\right] \\
& \cdot \exp \left[-n I\left(Y^{*}+W\right)\right] \exp \left[-\sqrt{n}\left\langle A^{-1}\left(Y^{*}+W\right), X\right\rangle\right] d P_{A_{\perp}}(X) d z
\end{aligned}
$$

By (5.39), $d P_{A_{\perp}}(X)=\tilde{\Delta}^{-1 / 2} \exp \left((1 / 2)\left\langle F^{\prime \prime}\left(Y^{*}\right) X, X\right\rangle\right) d P_{B_{\perp}}(X)$. Comparing (5.48) with (5.44), we see that we are done once we have shown for all $X \in S_{n}(\delta, z)$

$$
\begin{aligned}
n F\left(Y^{*}+\right. & W+X / \sqrt{n})-(n / 2)\left\langle F^{\prime \prime}\left(Y^{*}\right) X / \sqrt{n}, X / \sqrt{n}\right\rangle \\
& +n I\left(Y^{*}+W\right)+n\left\langle A^{-1}\left(Y^{*}+W\right), X / \sqrt{n}\right\rangle \\
= & n G\left(Y^{*}+W\right)+n \tilde{F}_{3}(W, X / \sqrt{n}) .
\end{aligned}
$$

But by the definitions of $G$ and $\tilde{F}_{3},(5.49)$ follows if we prove for all $X \in \mathscr{K}^{\perp}$

$$
\left\langle F^{\prime}\left(Y^{*}+W\right), X\right\rangle+\left\langle A^{-1}\left(Y^{*}+W\right), X\right\rangle=0 .
$$

But this follows from (5.4) in Lemma 5.1 if in the latter we write $z / \sqrt{n}$ for $z$. This proves (5.8). 
Proof of Lemma 5.4. Let $Y$ be an $\mathcal{F}$-valued random variable with distribution $P_{A}$. Define $h(\tau Y ; \cdot)$ to be the conditional distribution of $z(Y)$ given $\tau Y$ and $P$ the marginal distribution of $\tau Y$. By [Skorohod, p. 63], we have

$$
\int f(z(Y) U, \tau Y) d P_{A}=\int_{\mathbf{R} \times \mathcal{K}^{\perp}} f(z U, X) d h(X ; z) d P(X) .
$$

We first calculate $h(\tau Y ; \cdot)$ and $P$. We then prove $P \ll P_{A_{\perp}}$ and calculate $d P / d P_{A_{\perp}}$ to go from (5.51) to (5.43).

We define

$$
\mu(\tau Y):=E_{P_{A}}\{z(Y) \mid \tau Y\}, \quad \sigma^{2}:=E_{P_{A}}\{z((Y)-\mu)\}^{2} .
$$

Then we have (cf. [Simon, Theorem 3.9])

$$
d h(\tau Y ; z)=\frac{1}{\sqrt{2 \pi \sigma^{2}}} \exp \left[-(z-\mu(\tau Y))^{2} / 2 \sigma^{2}\right] d z
$$

The conditional mean $\mu(\tau Y)$ is the unique functional of the form $\langle W, \tau Y\rangle$ for some element $W \in \mathcal{H}$ which satisfies for any $V \in \mathcal{H}$

$$
E_{P_{A}}\{(z(Y)-\mu(\tau Y))\langle\tau Y, V\rangle\}=0 .
$$

Using $z(Y):=\langle Y, U\rangle, \quad \tau Y:=Y-\langle Y, U\rangle U, E_{P_{A}}\left\{\left\langle V_{1}, Y\right\rangle\left\langle V_{2}, Y\right\rangle\right\}=\left\langle A V_{1}, V_{2}\right\rangle$, where $V_{1}, V_{2}$ are arbitrary elements of $\mathcal{H}$, one verifies that

$$
\mu(\tau Y)=-\left\langle A^{-1} U, \tau Y\right\rangle /\left\langle A^{-1} U, U\right\rangle, \quad \sigma^{2}=1 /\left\langle A^{-1} U, U\right\rangle .
$$

From (5.52), we have for $X \in \mathscr{K}^{\perp}$

$d h(X ; z)=\left(\frac{\left\langle A^{-1} U, U\right\rangle}{2 \pi}\right)^{1 / 2} \exp \left[-I(z U)-z\left\langle A^{-1} U, X\right\rangle\right] \exp \left[-\frac{\left\langle A^{-1} U, X\right\rangle^{2}}{2\left\langle A^{-1} U, U\right\rangle}\right] d z$.

We now find $P$. The random variable $\tau Y$ has mean zero. For any elements $V_{1}$, $V_{2} \in \mathcal{H}$

$$
E_{P_{A}}\left\{\left\langle V_{1}, \tau Y\right\rangle\left\langle V_{2}, \tau Y\right\rangle\right\}=E_{P_{A}}\left\{\left\langle\tau V_{1}, Y\right\rangle\left\langle\tau V_{2}, Y\right\rangle\right\}=\left\langle\tau A \tau V_{1}, V_{2}\right\rangle .
$$

Thus, $\tau Y$ has covariance operator $\tau A \tau$ and we write $P=P_{\tau A \tau}$.

By (5.51), (5.53), we are finished once we prove for $X \in \mathscr{K}^{\perp}$

$$
d P_{\tau A \tau}(X)=\left[\langle A U, U\rangle\left\langle A^{-1} U, U\right\rangle\right]^{-1 / 2} \exp \left[\frac{\left\langle A^{-1} U, X\right\rangle^{2}}{2\left\langle A^{-1} U, U\right\rangle}\right] d P_{A_{\perp}} .
$$

We prove (5.54) by showing

$$
d P_{A_{\perp}}=\left[\langle A U, U\rangle\left\langle A^{-1} U, U\right\rangle\right]^{1 / 2} \exp \left[-\frac{\left\langle A^{-1} U, X\right\rangle^{2}}{2\left\langle A^{-1} U, U\right\rangle}\right] d P_{\tau A \tau}
$$

We derive (5.55) from Lemma 4.4. Define $\mathcal{H}_{1}:=\mathcal{K}^{\perp}, A_{1}:=\tau A \tau$,

$$
\Lambda X:=\left(\left\langle\tau A^{-1} U, X\right\rangle /\left\langle A^{-1} U, U\right\rangle\right) \tau A^{-1} U
$$

for $X \in \mathcal{K}^{\perp}, B_{1}:=A_{\perp}:=\tau \rho A \rho \tau$. We must prove

$$
A_{1}^{-1}+\Lambda>0 \text { on } \mathscr{D}\left(A_{1}^{-1}\right):=\mathscr{K}^{\perp} \cap \mathscr{D}\left(A^{-1}\right),
$$




$$
\begin{gathered}
B_{1}=\left(A_{1}^{-1}+\Lambda\right)^{-1}, \\
\Delta_{1}:=\operatorname{det}\left(I+A_{1} \Lambda\right)=\langle A U, U\rangle\left\langle A^{-1} U, U\right\rangle .
\end{gathered}
$$

We prove (5.57). Define the bounded operator $\bar{\rho}$ on $\mathcal{H}$ by

$$
\bar{\rho} Y:=Y-\left[\left\langle A^{-1} U, Y\right\rangle /\left\langle A^{-1} U, U\right\rangle\right] U .
$$

After straightforward modifications in the proof of Lemma 5.2 (formally, replacing $A$ by $A^{-1}$ and $A^{-1}$ by $A$ ), one finds $A_{1}^{-1}=\tau \bar{\rho} A^{-1} \bar{\rho} \tau$. Since $\tau \bar{\rho}=\tau$, we have for $X \in \mathscr{D}\left(A_{1}^{-1}\right)$

$$
A_{1}^{-1} X=\tau A^{-1} \bar{\rho} \tau X=\tau A^{-1} \tau X-\frac{\left\langle A^{-1} U, \tau X\right\rangle}{\left\langle A^{-1} U, U\right\rangle} \tau A^{-1} U=\tau A^{-1} \tau X-\Lambda X .
$$

Thus for $X \in \mathscr{D}\left(A_{1}^{-1}\right),\left(A_{1}^{-1}+\Lambda\right) X=\tau A^{-1} \tau X$. This proves (5.57) and since, by Lemma 5.2, $B_{1}:=A_{\perp}=\left(\tau A^{-1} \tau\right)^{-1}$, (5.58) also follows. We now prove (5.59). We have $\Delta_{1}=\operatorname{det}\left(I+\sqrt{A_{1}} \Lambda \sqrt{A_{1}}\right)$ and $\sqrt{A_{1}} \Lambda \sqrt{A_{1}}$ is the rank one operator on $\mathcal{K}^{\perp}$ given by

$$
\sqrt{A_{1}} \Lambda \sqrt{A_{1}} X=\frac{\left\langle\tau A^{-1} U, \sqrt{\tau A \tau} X\right\rangle}{\left\langle A^{-1} U, U\right\rangle} \sqrt{\tau A \tau} \tau A^{-1} U
$$

Thus $\Delta_{1}=1+\left\langle\tau A \tau A^{-1} U, A^{-1} U\right\rangle /\left\langle A^{-1} U, U\right\rangle$. Using $\tau Y:=Y-\langle Y, U\rangle U$, one gets (5.59) after a short calculation.

We now turn to the probabilistic limit theorems. We first prove Theorem 2.7, then state and prove extensions of Theorems 2.5 and 2.7 to the case where $G$ has nonunique, isolated minimum points of which some are simply degenerate and the rest nondegenerate. These extensions are given in Theorem 5.5. We prove Theorems 2.7 and 5.5 by applying Lemmas 4.1, 4.2, and 5.3 as modified by Remarks 4.1(a), 4.2(a), and 5.3(a) respectively.

Proof of Theorem 2.7. We prove that for any $\phi \in C(\mathcal{H})$

$$
\frac{\int \phi\left(\left(Y-\sqrt{n} Y^{*}\right) / n^{1 / 2-x}\right) \exp [-n F(Y / \sqrt{n})] d P_{A}(Y)}{\int \exp [-n F(Y / \sqrt{n})] d P_{A}(Y)} \rightarrow \frac{\int_{\mathbf{R}} \phi(z U) e^{-\lambda z^{k}} d z}{\int_{\mathbf{R}} e^{-\lambda z^{k}} d z},
$$

where we write $x$ for $1 / k$. We multiply the numerator and denominator of the left-hand side of $(5.60)$ by $n^{-(1 / 2-x)} \exp \left(n G^{*}\right)$. By Lemma 4.1 the resulting quotient equals $(\beta:=1 / 2-x)$

$$
\frac{n^{\beta} e^{n G^{*}} \int_{S\left(\sqrt{ } Y^{*}, n n \delta\right)} \phi\left(\left(Y-\sqrt{n} Y^{*}\right) / n^{\beta}\right) \exp [-n F(Y / \sqrt{n})] d P_{A}+O\left(e^{-n c}\right)}{n^{\beta} e^{n G^{*}} \int_{S\left(, n Y^{*}, v n \delta\right)} \exp [-n F(Y / \sqrt{n})] d P_{A}+O\left(e^{-n c}\right)}
$$

for any $\delta>0$ and $c=c(\delta)>0$. We apply Lemma 5.3 to the numerator and denominator of (5.52). The integral in the numerator of (5.61) equals the integral on the left-hand side of (5.8) if in the latter we pick

$$
\psi(Y):=\phi\left(n^{-(1 / 2-x)}\left(\sqrt{n} Y-\sqrt{n} Y^{*}\right)\right)=\phi\left(n^{x}\left(Y-Y^{*}\right)\right) .
$$


Hence for $\delta \in(0, \bar{\delta}](5.61)$ equals

$$
\frac{\int_{|z|<\delta n^{x}} \int_{S(\delta, n, z)} \phi\left(z U+n^{x} \Phi\left(z U / n^{x}\right)+X / n^{\beta}\right) e^{-n\left(H_{n}+\tilde{F}_{3, n}\right)} d \tilde{T}(X, z)+O\left(e^{-n c}\right)}{\int_{|z|<\delta n^{x}} \int_{S(\delta, n, z)} e^{-n\left(H_{n}+\tilde{F}_{3, n}\right)} d \tilde{T}(X, z)+O\left(e^{-n c}\right)},
$$

where

$$
\begin{gathered}
H_{n}:=G\left(Y^{*}+W\left(z / n^{x}\right)\right)-G\left(Y^{*}\right)-\lambda\left(z / n^{x}\right)^{k} \\
\tilde{F}_{3, n}:=\tilde{F}_{3}\left(W\left(z / n^{x}\right), X / \sqrt{n}\right) \text { and } d \tilde{T}(X, z):=d P_{B_{\perp}}(X) e^{-\lambda z^{k}} d z .
\end{gathered}
$$

We want to use the dominated convergence theorem to determine the $n \rightarrow \infty$ limits of the integrals in (5.63). By (5.32), given $b_{1}>0$ there exists $\delta \in(0, \bar{\delta}]$ such that if $z$ satisfies $|z|<\delta n^{x}$, then

$$
\left|n H_{n}\right| \leqslant b_{1} z^{k} \text {. }
$$

By (5.23), given $b_{2}>0$ there exists $\delta \in(0, \bar{\delta}]$ such that if $z$ satisfies $|z|<\delta n^{x}$ and $X \in S(\delta, n, z)$, then

$$
\left|n \tilde{F}_{3, n}\right| \leqslant b_{2}\|X\|^{2}
$$

For $\psi$ equal to $\phi$ or 1 , define

$$
\psi_{n}:=\psi\left(z U+n^{x} \Phi\left(z U / n^{x}\right)+X / n^{1 / 2-x}\right) .
$$

By (5.64), (5.65), if $\delta \in(0, \bar{\delta}]$ is sufficiently small, then

$$
\left|\psi_{n}\right| e^{-n H_{n}} e^{-n \tilde{F}_{3, n}} \leqslant \text { const } \exp \left[b_{1} z^{k}+b_{2}\|X\|^{2}\right] .
$$

By Lemma 4.3, the latter exponential is integrable with respect to $d P_{B_{\perp}}(X) e^{-\lambda z^{k}} d z$ over $\mathcal{K}^{\perp} \times \mathbf{R}$ provided $b_{1}$ and $b_{2}$ are sufficiently small. But $b_{1}$ and $b_{2}$ small imply that $\delta$ is small. We conclude that for sufficiently small $\delta \in(0, \bar{\delta}]$ we may use the dominated convergence theorem to determine the limits of the integrals in (5.63). For fixed $z \in \mathbf{R}, n^{x} \Phi\left(z U / n^{x}\right) \rightarrow 0$ as $n \rightarrow \infty$ by (5.3). Hence for fixed $z \in \mathbf{R}$ and $X \in \mathcal{K}^{\perp}, \psi_{n} \rightarrow \psi(z U), n H_{n} \rightarrow 0, n \tilde{F}_{3, n} \rightarrow 0$; also $\chi_{\left(-\delta n^{x}, \delta n^{x}\right)}(z) \rightarrow 1, \chi_{S(\delta, n, z)}(X) \rightarrow 1$. We conclude that as $n \rightarrow \infty$ the quotient in (5.63) equals

$$
\frac{\int_{\mathbf{R}} \phi(z U) e^{-\lambda z^{k}} d z+o(1)+O\left(e^{-n c}\right)}{\int_{\mathbf{R}} e^{-\lambda z^{k}} d z+o(1)+O\left(e^{-n c}\right)}=\frac{\int_{\mathbf{R}} \phi(z U) e^{-\lambda z^{k}} d z}{\int_{\mathbf{R}} e^{-\lambda z^{k}} d z}+o(1) .
$$

This proves (5.60).

We now assume that $G$ has nonunique minimum points $\left\{Y_{\alpha}^{*} ; \alpha=1, \ldots, L\right\}$ of which the first $J$ are simply degenerate and of finite type $(J \in\{1, \ldots, L\})$ and the last $L-J$ nondegenerate. Thus we have $L \in\{2,3, \ldots\}$. We extend Theorems 2.5 and 2.7 to cover this case. The following quantities arise in these extensions. For each $\alpha \in\{1, \ldots, J\}$, we pick a unit vector $U_{\alpha} \in \mathscr{K}_{\alpha}:=\operatorname{ker}\left\{G^{\prime \prime}\left(Y_{\alpha}^{*}\right)\right\}$. Let $\tau_{\alpha}$ be the orthogonal projection onto $\mathcal{K}_{\alpha}^{\perp}$. We define the operator $\rho_{\alpha} \in \mathscr{B}(\mathcal{H})$ by

$$
\rho_{\alpha} Y:=Y-\left[\left\langle A U_{\alpha}, Y\right\rangle /\left\langle A U_{\alpha}, U_{\alpha}\right\rangle\right] U_{\alpha}
$$

and the determinant

$$
\tilde{\Delta}_{\alpha}:=\operatorname{det}\left[I+\tau_{\alpha} \rho_{\alpha} A \rho_{\alpha} \tau_{\alpha} F^{\prime \prime}\left(Y_{\alpha}^{*}\right) \tau_{\alpha}\right]
$$


$\tilde{\Delta}_{\alpha}$ is well defined and $\tilde{\Delta}_{\alpha}>0$ by Lemma 5.3. We let $k_{\alpha}$ and $\lambda_{\alpha}$ denote the type and strength, respectively, of $Y_{\alpha}^{*}$ and define

$$
k^{\#}:=\max \left\{k_{\alpha}: \alpha=1, \ldots, L\right\} .
$$

The number $k^{\#}$ is called the maximal type. Finally, we define numbers

$$
\bar{b}_{\alpha}:=\left\{\begin{array}{l}
{\left[2 \pi\left\langle A U_{\alpha}, U_{\alpha}\right\rangle \tilde{\Delta}_{\alpha}\right]^{-1 / 2} \int_{\mathbf{R}} \exp \left[-\lambda_{\alpha} z^{k_{\alpha}}\right] d z \text { if } k_{\alpha}=k^{\sharp},} \\
0 \quad \text { if } k_{\alpha}<k^{\#},
\end{array}\right.
$$

and

$$
b_{\alpha}:=\overline{b_{\alpha}} / \sum_{\beta=1}^{J} \overline{b_{\beta}} \text {. }
$$

In order to simplify the statement of Theorem 5.5, we define the type of the nondegenerate minimum points, if any, to be two; i.e., if $J<L$, then $k_{\alpha}:=2$ for $\alpha \in\{J+1, \ldots, L\}$. Since $G$ is assumed to have simply degenerate minimum points, we see that for $\alpha \in\{J+1, \ldots, L\}, k_{\alpha}=2<k^{\sharp}$. For such $\alpha$, we define $\vec{b}_{\alpha}:=0$, $b_{\alpha}:=0$.

In the next theorem (5.74) generalizes Theorem 2.5 and (5.74)-(5.75) generalize Theorem 2.7. We recall that $\left\{Y_{n}\right\}$ are $\mathcal{G}$-valued random variables with distributions $\left\{Q_{n}\right\}$ in (1.6). The symbol $\stackrel{0}{\rightarrow}$ in the line after (5.75) denotes vague convergence (defined just before Theorem 4.6). See (2.28) and the statement of Theorem 2.7 for the definition of the measures $\left\{\xi_{k_{\alpha}, \lambda_{\alpha}}\right\}$ on $\mathbf{R}$ and $\left\{\xi_{k_{\alpha}, \lambda_{\alpha}}^{U_{\alpha}}\right\}$ on $\mathcal{H}$ which appear in (5.76) and the next line.

THEOREM 5.5. Suppose that $G$ has minimum points $\left\{Y_{\alpha}^{*} ; \alpha=1, \ldots, L\right\}$ of which the first $J$ are simply degenerate and of finite type $(J \in\{1, \ldots, L\})$ and the rest nondegenerate. Suppose that $F$ satisfies the bound (2.1)(b). Then for all $\phi \in C(\mathcal{H C})$

$$
\frac{\int \phi(Y / n) \exp (-n F(Y / n)) d P_{A}(Y / \sqrt{n})}{Z_{n}} \rightarrow \sum_{\left\{\alpha: k_{\alpha}=k^{\sharp}\right\}} b_{\alpha} \phi\left(Y_{\alpha}^{*}\right) ;
$$

i.e., $Y_{n} / \stackrel{\stackrel{D}{\rightarrow}}{\rightarrow} \Sigma_{\left\{\alpha: k_{\alpha}=k^{\sharp}\right\}} b_{\alpha} \delta_{Y_{\alpha}^{*}}$.For each $\alpha \in\{1, \ldots, J\}$ for which $k_{\alpha}=k^{\sharp}$ and for all $\phi \in C(\mathcal{H C})$ which vanish at infinity,

$$
\frac{\int \phi\left(\left(Y-n Y_{\alpha}^{*}\right) / n^{1-1 / k_{\alpha}}\right) \exp (-n F(Y / n)) d P_{A}(Y / \sqrt{n})}{Z_{n}} \rightarrow b_{\alpha} \int_{\mathbf{R}} \phi\left(z U_{\alpha}\right) d \xi_{k_{\alpha}, \lambda_{\alpha}}(z) ;
$$

i.e., $\left(Y_{n}-n Y_{\alpha}^{*}\right) / n^{1-1 / k_{\alpha}} \stackrel{v}{\rightarrow} b_{\alpha} \xi_{k_{\alpha}, \lambda_{\alpha}}^{U_{\alpha}}$. If there exists a unique index $\alpha($ say $\alpha=1)$ such that $k_{\alpha}=k^{\sharp}$, then (5.75) can be strengthened: for all $\phi \in C(\mathcal{F C})$

$$
\frac{\int \phi\left(\left(Y-n Y_{\alpha}^{*}\right) / n^{1-1 / k_{\alpha}}\right) \exp (-n F(Y / n)) d P_{A}(Y / \sqrt{n})}{Z_{n}} \rightarrow \int_{\mathbf{R}} \phi\left(z U_{1}\right) d \xi_{k_{1}, \lambda_{1}}(z) ;
$$

i.e., $\left(Y_{n}-n Y_{1}^{*}\right) / n^{1-1 / k_{1}} \stackrel{\mathscr{D}}{\rightarrow} \xi_{k_{1}, \lambda_{1}}^{U_{1}}$. 
Proof of Theorem 5.5. To prove (5.74), we prove that for any $\phi \in C(\mathcal{H})$

$$
\frac{\int \phi(Y / \sqrt{n}) \exp [-n F(Y / \sqrt{n})] d P_{A}(Y)}{\int \exp [-n F(Y / \sqrt{n})] d P_{A}(Y)} \rightarrow \sum_{\left\{\alpha: k_{\alpha}=k^{\sharp}\right\}} b_{\alpha} \phi\left(Y_{\alpha}^{*}\right)
$$

as $n \rightarrow \infty$. Let $\Sigma(\alpha, \delta):=S\left(\sqrt{n} Y_{\alpha}^{*}, \sqrt{n} \delta\right)$. We multiply the numerator and denominator of the left-hand side of $(5.77)$ by $n^{-\left(1 / 2-x^{\sharp}\right)} \exp \left(n G^{*}\right)$, where $x^{\#}:=1 / k^{\#}$. By Lemma 4.1, the resulting quotient equals

$$
\frac{\sum_{\alpha=1}^{L} n^{-\left(1 / 2-x^{\sharp}\right)} e^{n G^{*}} \int_{\Sigma(\alpha, \delta)} \phi(Y / \sqrt{n}) \exp [-n F(Y / \sqrt{n})] d P_{A}(Y)+O\left(e^{-n c}\right)}{\sum_{\alpha=1}^{L} n^{-\left(1 / 2-x^{\sharp}\right)} e^{n G^{*}} \int_{\Sigma(\alpha, \delta)} \exp [-n F(Y / \sqrt{n})] d P_{A}(Y)+O\left(e^{-n c}\right)}
$$

for any $\delta>0$ so small that $\Sigma(\alpha, \delta) \cap \Sigma(\beta, \delta)=\varnothing$ if $\alpha \neq \beta$ and $c=c(\delta)>0$.

We first consider the summands in (5.78) with $\alpha \in\{1, \ldots, J\}$, which correspond to the simply degenerate minimum points $\left\{Y_{\alpha}^{*} ; \alpha=1, \ldots, J\right\}$. We apply Lemma 5.3 to each of these terms. There exists $\bar{\delta}>0$ such that for all $\delta \in(0, \bar{\delta}]$

$$
\begin{aligned}
& n^{-\left(1 / 2-x^{\sharp}\right)} e^{n G^{*}} \int_{\Sigma(\alpha, \delta)} \psi(Y / \sqrt{n}) \exp [-n F(Y / \sqrt{n})] d P_{A}(Y) \\
& =\gamma_{\alpha} n^{x^{\sharp}-x_{\alpha}} \int_{|z|<\delta n^{x_{\alpha}}} \int_{S_{\alpha}(\delta, n, z)} \bar{\psi}_{\alpha, n} e^{-n H_{\alpha, n}} e^{-n \tilde{F}_{3, \alpha, n}} d P_{B_{\perp, \alpha}}(X) \exp \left(-\lambda_{\alpha} z^{k_{\alpha}}\right) d z
\end{aligned}
$$

where $\gamma_{\alpha}:=\left[(2 \pi)\left\langle A U_{\alpha}, U_{\alpha}\right\rangle \tilde{\Delta}_{\alpha}\right]^{-1 / 2}, S_{\alpha}(\delta, n, z):=S\left(Y_{\alpha}^{*} ; \delta, n, z\right)$,

$$
\bar{\psi}_{\alpha, n}:=\psi\left(Y_{\alpha}^{*}+W_{\alpha}\left(z / n^{x_{\alpha}}\right)+X / \sqrt{n}\right)
$$

$\psi$ equals $\phi$ or $1, W_{\alpha}:=W\left(Y_{\alpha}^{*} ; \cdot\right)$,

$$
H_{\alpha}:=G\left(Y_{\alpha}^{*}+W_{\alpha}\left(z / n^{x_{\alpha}}\right)\right)-G\left(Y_{\alpha}^{*}\right)-\lambda_{\alpha}\left(z / n^{x_{\alpha}}\right)^{k_{\alpha}},
$$

$\tilde{F}_{3, \alpha, n}:=\tilde{F}_{3}\left(Y_{\alpha}^{*} ; W_{\alpha}\left(z / n^{x_{\alpha}}\right), X / \sqrt{n}\right)$, and $P_{B_{\perp, \alpha}}:=P_{B_{\perp}\left(Y_{\alpha}^{*}\right)}$. As in the proof of Theorem 2.7, we may use the dominated convergence theorem to determine the $n \rightarrow \infty$ limit of the iterated integral on the right-hand side of (5.79). Since, as $n \rightarrow \infty, \bar{\psi}_{\alpha, n} \rightarrow \psi\left(Y_{\alpha}^{*}\right)$ for fixed $z$ real, $X \in \mathcal{K}^{\perp}$, this iterated integral tends to $\psi\left(Y_{\alpha}^{*}\right) \int_{\mathbf{R}} \exp \left[-\lambda_{\alpha} z^{k_{\alpha}}\right] d z$. Since $b_{\alpha}=\gamma_{\alpha} \int_{\mathbf{R}} \exp \left[-\lambda_{\alpha} z^{k_{\alpha}}\right] d z$, we conclude that for $\alpha \in$ $\{1, \ldots, J\}$

$$
\begin{aligned}
n^{-\left(1 / 2-x^{*}\right)} e^{n G^{*}} \int_{\Sigma(\alpha, \delta)} \psi(Y / \sqrt{n}) \exp [ & -n F(Y / \sqrt{n})] d P_{A}(Y) \\
& =b_{\alpha} n^{x^{*}-x_{\alpha}}\left[\psi\left(Y_{\alpha}^{*}\right)+o(1)\right] \text { as } n \rightarrow \infty .
\end{aligned}
$$

Assuming $J<L$, we now consider the summands in (5.78) with $\alpha \in\{J+1, \ldots, L\}$, which correspond to the nondegenerate minimum points $\left\{Y_{\alpha}^{*} ; \alpha=J+1, \ldots, L\right\}$. By the proof of (4.41) in Theorem 4.6, we have for such $\alpha$

$$
\begin{aligned}
n^{-\left(1 / 2-x^{*}\right)} e^{n G^{*}} \int_{\Sigma(\alpha, \delta)} \psi(Y / \sqrt{n}) \exp [-n F(Y / \sqrt{n})] d P_{A}(Y) \\
=n^{x^{*}-1 / 2}\left[\psi\left(Y_{\alpha}^{*}\right)+o(1)\right] \text { as } n \rightarrow \infty
\end{aligned}
$$


We substitute (5.82)-(5.83) into (5.78) and take $n \rightarrow \infty$. Since $\frac{1}{2}>x_{\alpha} \geqslant x^{\#}$ for $\alpha \in\{1, \ldots, J\}$, we obtain (5.77).

We next prove (5.76) assuming that $\alpha=1$ is the unique index for which $k_{\alpha}=k^{\#}$. For $\alpha \in\{2, \ldots, J\}$, we have that $2<k_{\alpha}<k^{\#}$ and $Y_{\alpha}^{*}$ is simply degenerate while for $\alpha \in\{J+1, \ldots, L\}$, we have that $k_{\alpha}=2$ and $Y_{\alpha}^{*}$ is nondegenerate. We multiply the numerator and denominator of the left-hand side of (5.76) by $n^{-\left(1 / 2-x^{*}\right)} e^{n G^{*}}$, where $x^{\#}:=1 / k^{\#}$. By Lemma 4.1 , the resulting quotient is given by an expression as in (5.78) but with $\phi(Y / \sqrt{n})$ replaced by $\phi\left(n^{-\left(1 / 2-x^{\sharp}\right)}\left(Y-\sqrt{n} Y_{1}^{*}\right)\right)$. We apply Lemma 5.3 to the summands in this expression with $\alpha \in\{1, \ldots, J\}$ and Lemma 4.2 to the summands with $\alpha \in\{J+1, \ldots, L\}$. Arguing as above but omitting details, we find that the left-hand side of (5.76) equals

$$
\frac{\int_{\mathbf{R}} \phi\left(z U_{1}\right) \exp \left(-\lambda_{1} z^{k_{1}}\right) d z+o(1)+\sum_{\alpha=2}^{L} O\left(n^{x^{\sharp}-x_{\alpha}}\right)+O\left(e^{-n c}\right)}{\int_{\mathbf{R}} e^{-\lambda_{1} z^{k}} d z+o(1)+\sum_{\alpha=2}^{L} O\left(n^{x^{\sharp}-x_{\alpha}}\right)+O\left(e^{-n c}\right)},
$$

where $x_{\alpha}:=1 / k_{\alpha}$. As $n \rightarrow \infty$, this tends to the right-hand side of (5.76) since $x_{\alpha}>x^{\#}$ for $\alpha \in\{2, \ldots, L\}$.

We next prove (5.75) for $\alpha=1$. Arguing as above but omitting details, we find that for some $\bar{\delta}>0, \delta \in(0, \bar{\delta}], c=c(\delta)>0$ the left-hand side of (5.75) equals

$$
\begin{aligned}
& {\left[\gamma_{1} \int_{\mathbf{R}} \phi\left(z U_{1}\right) \exp \left(-\lambda_{1} z^{k_{1}}\right) d z+o(1)\right.} \\
& +\sum_{\left\{\alpha: \alpha \neq 1, k_{\alpha}=k^{*}\right\}} \int_{|z|<\delta n^{x}} \int_{S_{\alpha}(\delta, n, z)} \phi\left(n^{x}\left(Y_{\alpha}^{*}-Y_{1}^{*}\right)+z U_{\alpha}+n^{x} \Phi\left(z U_{1} / n^{x}\right)+X / n^{1 / 2-x}\right) \\
& \left.\cdot e^{-n H_{\alpha, n}} e^{-n \tilde{F}_{3, \alpha, n}} d P_{B_{\perp, \alpha}} \exp \left(-\lambda_{\alpha} z^{k_{\alpha}}\right) d z+\sum_{\left\{\alpha: k_{\alpha}<k^{\sharp}\right\}} O\left(n^{x-x_{\alpha}}\right)+O\left(e^{-n c}\right)\right] / T_{n},
\end{aligned}
$$

where

$$
T_{n}:=\sum_{\left\{\beta: k_{\beta}=k^{\sharp}\right\}} \bar{b}_{\beta}+o(1)+\sum_{\left\{\beta: k_{\beta}<k^{\sharp}\right\}} O\left(n^{x-x_{\beta}}\right)+O\left(e^{-n c}\right)
$$

In (5.85), we write $x$ for $1 / k^{\#}$ but otherwise the notation is the same as in (5.79). Since $\phi$ is bounded and vanishes at infinity, we see by dominated convergence that the integrals in (5.85) corresponding to $\alpha \neq 1, k_{\alpha}=k^{\#}$, tend to zero as $n \rightarrow \infty$ provided $\delta$ is sufficiently small. Thus, as $n \rightarrow \infty,(5.85)$ tends to

$$
\gamma_{1} \int_{-\infty}^{\infty} \phi\left(z U_{1}\right) \exp \left(-\lambda_{1} z^{k_{1}}\right) d z / \sum_{\left\{\beta: k_{\beta}=k^{\sharp}\right\}} \overline{b_{\beta}}
$$

This equals the right-hand side of (5.75) since $\bar{b}_{1}=\gamma_{1} \int_{\mathbf{R}} \exp \left(-\lambda_{1} z^{k_{1}}\right) d z$ and $b_{1}=$ $\bar{b}_{1} / \Sigma_{\left\{\beta: k_{\beta}=k^{\sharp}\right\}} \bar{b}_{\beta}$. 


\section{REFERENCES}

M. S. Berger, Nonlinearity and functional analysis, Academic Press, New York and London, 1977.

S. Coleman, The uses of instantons, Lectures delivered at the 1977 International School of Subnuclear Physics, Ettore Majorana.

M. D. Donsker and S. R. S. Varadhan, Asymptotic evaluation of certain Markov process expectations for large time. III, Comm. Pure Appl. Math. 29 (1976), 389-461.

R. S. Ellis and C. M. Newman, The statistics of Curie-Weiss models, J. Statist. Phys. 19 (1978), 149-161.

R. S. Ellis and J. S. Rosen, (1) Asymptotics of certain random fields on a circle. Proc. Colloq. Random Fields: Rigorous Results in Statistical Mechanics and Quantum Field Theory, Esztergom, Hungary (June, 1979). Ser. Colloq. Math. Soc. Jànos-Bolyai, vol. 27, North-Holland, Amsterdam, pp. 279-321.

(2) L_Laplace's method for Gaussian integrals with an application to statistical mechanics, Ann. Probab. 10 (1982), 47-66. (Also see Erratum (to appear).)

A. Erdélyi, Asymptotics expansions, Dover, New York, 1956.

M. I. Freidlin, The action functional for a class of stochastic processes, Theor. Probab. Appl. 17 (1972), 511-515.

I. I. Gihman and A. V. Skorohod, The theory of stochastic processes. I, transl. by S. Kotz, Springer-Verlag, Berlin and New York, 1974.

I. C. Gohberg and M. G. Krein, Introduction to the theory of linear nonselfadjoint operators, transl. by A. Feinstein, Transl. Math. Monos., vol. 24, Amer. Math. Soc., Providence, R.I., 1970.

G. Kallianpur and H. Oodaira, Freidlin-Wentzell type estimates for abstract Wiener spaces, Sankhyā Ser. A 40 (1978), 116-137.

M. Pincus, Gaussian processes and Hammerstein integral equations, Trans. Amer. Math. Soc. 134 (1968), 193-216.

B. S. Rajput, The support of Gaussian measures on Banach spaces, Theor. Probab. Appl. 17 (1972), 728-734.

F. Riesz and B. Sz.-Nagy, Functional analysis, transl. by L. F. Boron, Ungar, New York, 1955.

M. Schilder, Some asymptotic formulas for Wiener integrals, Trans. Amer. Math. Soc. 125 (1966), 63-85.

L. S. Schulman, Techniques and applications of path integration, Wiley, New York and Toronto, 1981.

B. Simon, Integration and quantum physics, Academic Press, New York and London, 1979.

A. V. Skorohod, Integration in Hilbert space, transl. by K. Wickwire, Springer-Verlag, Berlin and New York, 1974.

N. M. Temme (editor), Nonlinear analysis. Vol. 2, Mathematisch Centrum, Amsterdam, 1976.

S. R. S. Varadhan, Asymptotic probabilities and differential equations, Comm. Pure Appl. Math. 19 (1966), 261-286.

A. D. Wentzell, Theorems on the action functional for Gaussian random functions, Theor. Probab. Appl. 17 (1972), 515-517.

F. W. Wiegel, Path integral methods in statistical mechanics, Phys. Rep. 16 (1975), 57-114.

Department of Mathematics and Statistics, University of Massachusetts, Amherst, MasSACHUSETTS 01003 